SANDIA REPORT

SAND98-2696

Unlimited Release

Printed December 1998

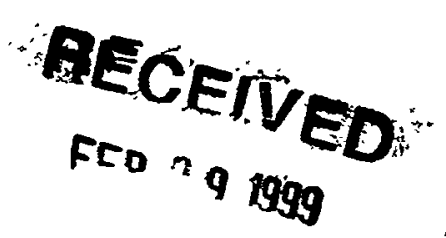

\title{
Gradient-Driven Diffusion of Multi-Atom Molecules Through Macromolecules and Membranes: LDRD 96-0021 Close-Out Report
}

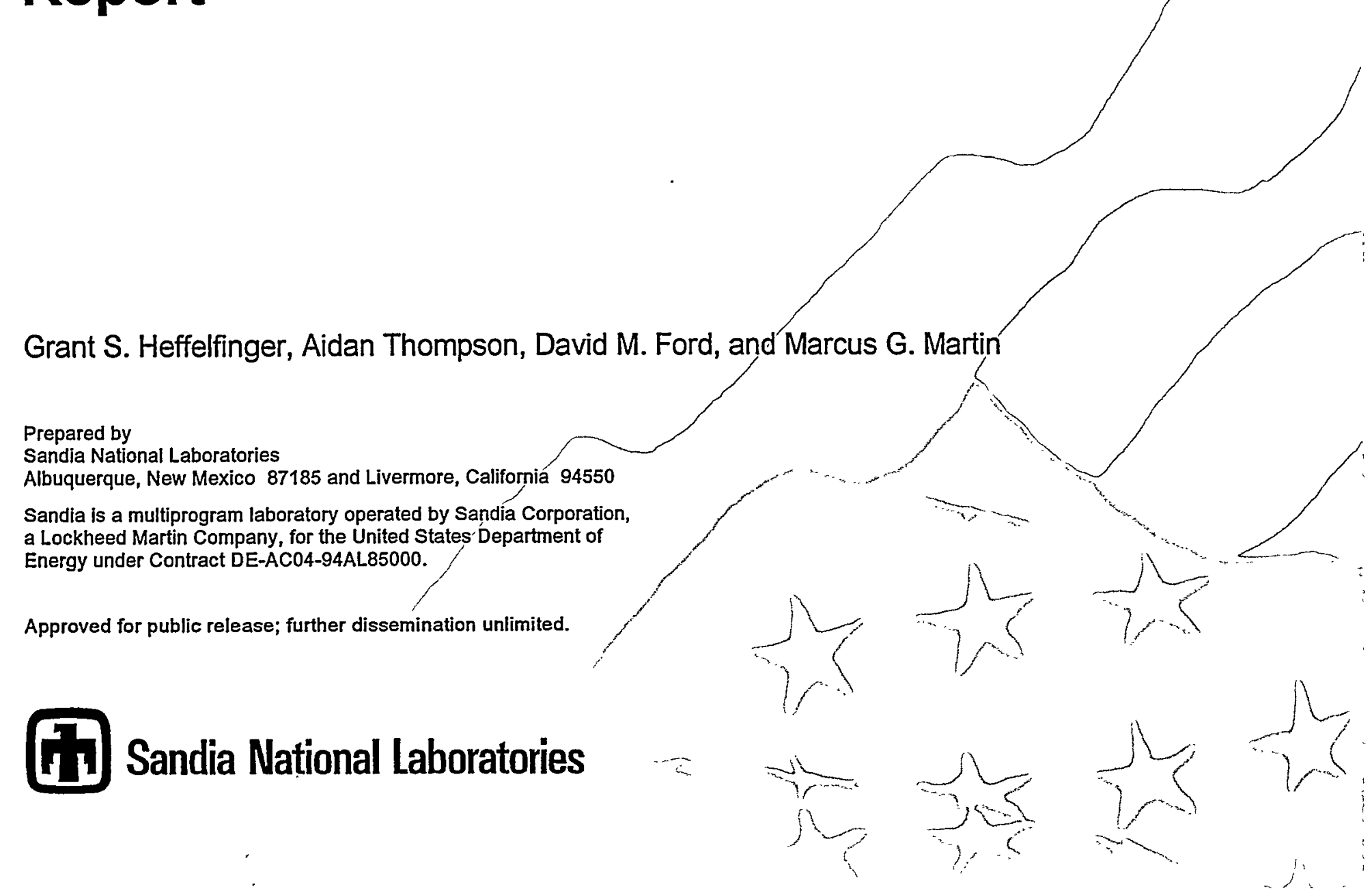


Issued by Sandia National Laboratories, operated for the United States Department of Energy by Sandia Corporation.

NOTICE: This report was prepared as an account of work sponsored by an agency of the United States Govermment. Neither the United States Government, nor any agency thereof, nor any of their employees, nor any of their contractors, subcontractors, or their employees, make any warranty, express or implied, or assume any legal liability or responsibility for the accuracy, completeness, or usefulness of any information, apparatus, product, or process disclosed, or represent that its use would not infringe privately owned rights. Reference herein to any specific commercial product, process, or service by trade name, trademark, manufacturer, or otherwise, does not necessarily constitute or imply its endorsement, recommendation, or favoring by the United States Government, any agency thereof, or any of their contractors or subcontractors. The views and opinions expressed herein do not necessarily state or reflect those of the United States Government, any agency thereof, or any of their contractors.

Printed in the United States of America. This report has been reproduced directly from the best available copy.

Available to DOE and DOE contractors from

Office of Scientific and Technical Information

P.O. Box 62

Oak Ridge, TN 37831

Prices available from (703) 605-6000

Web site: http://www.ntis.gov/ordering.htm

Available to the public from

National Technical Information Service

U.S. Department of Commerce

5285 Port Royal Rd

Springfield, VA 22161

NTIS price codes

Printed copy: A04

Microfiche copy: A01

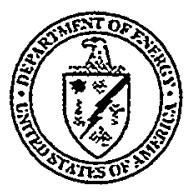




\section{DISCLAIMER}

Portions of this document may be illegible in electronic image products. Images are produced from the best available original document. 


\title{
Gradient-Driven Diffusion of Multi-Atom Molecules Through Macromolecules and Membranes: LDRD 96-0021 Close-out Report
}

\author{
Grant S. Heffelfinger and Aidan Thompson, \\ Materials Simulation Science Department \\ Sandia National Laboratories \\ P.O. Box 5800 \\ Albuquerque, NM 87185-1111 \\ David M. Ford, \\ Department of Chemical Engineering \\ Texas A\&M University \\ College Station, TX 778943-3122, \\ Marcus G Martin \\ Department of Chemistry \\ University of Minnesota \\ 207 Pleasant Street SE \\ Minneapolis, MN 55455-0431
}




\section{Abstract}

The goals of this Laboratory Directed Research \& Development (LDRD) effort were to develop and prototype a new molecular simulation method and companion parallel algorithm able to model diffusion of multi-atom molecules through macromolecules under conditions of a chemical potential gradient. At the start of the project, no such method existed, thus many important industrial and technological materials problems where . gradient-driven diffusion of multi-atom molecules is the predominant phenomenon were beyond the reach of molecular simulation (e.g. diffusion in polymers, a fundamental problem underlying polymer degradation in aging weapons).

This work built upon the previously developed dual control volume grand canonical molecular dynamics (DCV-GCMD) which enabled direct simulation of gradient-driven diffusion processes. DCV-GCMD was originally restricted to gradient-driven diffusion of small molecules and had been implemented in a code named LADERA. In this work, the method was extended first to enable the simulation of single-site species diffusing through a system comprised of multi-site (and single-site) species. Next, the method was generalized to allow the treatment of small multi-site species diffusing through multi-site species. Finally, a new implementation of the method, again in LADERA, was devised to enable the simulation of large, multi-site species diffusing through a multi-site system.

This report begins with an overview of the DCV-GCMD method for simulating diffusion in systems with chemical potential gradients, followed by a brief description of the parallel algorithm developed previously for the single-site implementation of the method and some examples of applications of the original method. The new parallel algorithm for DCVGCMD applied to single-site species diffusing through multi-site systems (e.g. uniatomic gases through macromolecules) is next presented and discussed followed by a prototype application of this capability. Finally, an extension of the DCV-GCMD method to diffusion of multi-site species through multi-site systems (e.g. polyatomic gas through macromolecules) is discussed followed by a novel algorithm to enable the simulation of gradient-driven diffusion of polyatomic species without employing insertions or deletions of the diffusing species. 


\section{Contents}

Summary vi

\section{Gradient-Driven Diffusion of Multi-Atom Molecules Through Mac- romolecules and Membranes: LDRD 96-0021 Close-out Report}

Introduction ........................................................................................................................ 1

Dual Control Volume Grand Canonical Molecular Dynamics (DCV-GCMD) ............. 2

Parallel DCV-GCMD for Single-Site Species Diffusing In Single-Site Systems ........... 4

Examples for Single-Site Species Diffusing In Single-Site Systems ............................... 5

Color Diffusion ............................................................................................................. 6

Uphill Diffusion ........................................................................................................... 6

Parallel DCV-GCMD for Single-Site Species Diffusing In Single-Site Systems ........... 7

The Parallel "GC Phase"................................................................................. 10

The Resulting Parallel DCV-GCMD Algorithm .......................................................... 13

Demonstration Calculations and Performance Characteristics.................................... 14

Parallel DCV-GCMD for Multi-Site Species in Multi-Site Systems .............................. 18

Dynamic Tag Reallocation ......................................................................................... 19

Elimination of Ghost Particles................................................................................ 20

Communication-free Energy Calculation .................................................................... 20

Molecular Insertion and Deletion ............................................................................ 21

The Resulting Parallel DCV-GCMD Algorithm ......................................................... 22

Chemical Potential Control for Large Molecules ............................................................ 23

Applications of the DCV-GCMD and Extended DCV-GCMD Methods ........................ 25

Diffusion in Polymers................................................................................................... 25

Constant Pressure DCV-GCMD ................................................................................ 28

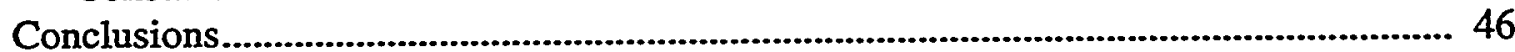

References........................................................................................................... 46

\section{Figures}

Figure 1. A schematic of a DCV-GCMD simulation.................................................... 3

Figure 2. Density profiles for a WCA bulk ternary experiencing uphill diffusion in component 2 ................................................................................................. 8

Figure 3. Chemical potentialprofilesfora WCAbulkternary experiencing uphill diffusion 9

Figure 4. CPU seconds per time step and number of reneighborings as functions of nghost, for system B1 with $r_{s}=0.5$ and $\Delta_{\text {sub }}=0.25$ on the nCUBE2 ......................... 16

Figure 5. CPU seconds per time step as a function of number of processors (a measure of system size)................................................................................................................ 18

Figure 6. Density profiles for all species in system B1 as a function of X-position...... 26

Figure 7. The central region of Figure 6 ...................................................................... 27

Figure 8. Density profiles for all species in system B2 as a function of $x$-position...... 28

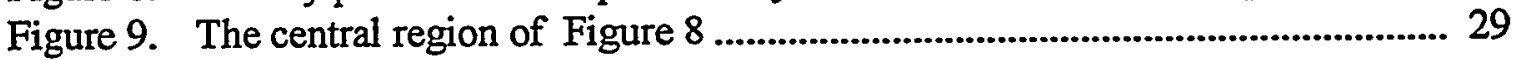


Figure 10. Plot of piston positions versus time for an NPT molecular dynamics simulation of a pure WCA fluid

Figure 11. Average flux profiles for the constant-volume DCV-GCMD simulation ..... 33

Figure 12. Time evolution of the concentration profile for component 2 in the constantpressure DCV-GCMD simulation.

Figure 13. Average concentration profiles sampled between timesteps 100,000 and 200,000 of the constant-pressure DCV-GCMD simulation.

Figure 14. Average flux profiles sampled between timesteps 100,000 and 200,000 of the constant-pressure DCV-GCMD simulation

Figure 15. Comparison of the average absolute flux profiles from the constant pressure color diffusion simulation with the results of the continuum model

Figure 16. Plot of diffusion fluxes and concentration gradients profiles in the gradient zone from the constant-pressure color diffusion simulation

Figure 17. Diffusion coefficient profiles for components 1 and 2 from the constantpressure color diffusion simulation.

Figure 18. Concentration profiles from simulations of gradient-driven diffusion in a binary mixture of WCA spheres with a 1:2 size ratio.

Figure 19. Flux profiles from simulations of gradient-driven diffusion in a binary mixture of WCA spheres with a 1:2 size ratio

Figure 20. Binary diffusion coefficient profiles from simulations of gradient-driven diffusion in a binary mixture of WCA spheres with a 1:2 size ratio

Figure 21. Maxwell-Stefan diffusion coefficient profiles from simulations of gradientdriven diffusion in a binary mixture of WCA spheres with a 1:2 size ratio.

\section{Tables}

Table 1. Uphill Diffusion Lennard-Jones Parameters and Diffusivities........................... 7

Table 2. Flux Data for System B1 .................................................................................. 26

Table 3. Flux Data for System B2 …................................................................... 29

Table 4. Constant-volume DCV-GCMD simulation results.............................................. 32

Table 5. Constant-pressure color diffusion continuum model parameters ...................... 39

Table 6. Constant-volume and constant pressure simulation results ............................... 42

Table 7. Thermodynamic conditions of binary diffusion simulations............................... 42 


\section{Summary}

That dual control volume grand canonical molecular dynamics is quickly becoming accepted as an extremely novel and useful simulation method is borne out by the rapidly growing number of researchers developing their own DCV-GCMD codes. Sandia's DCVGCMD code, LADERA, not only pioneered the DCV-GCMD method for modeling gradient-driven diffusion, but remains the leading DCV-GCMD code with several unique capabilities including: 1) the only massively parallel implementation of the method, 2) the only implementation of multi-atomic species diffusing through multi-atomic systems, 3) the only algorithm which enables the chemical potential control without the difficult and costly insertion and deletion of large diffusing species.

In this report, the rapidly evolving literature of classical simulations of chemical potential gradients is reviewed in the context of Sandia's multi-year effort on the LADERA DCVGCMD code and the work of the three-year Laboratory Directed Research and Development effort is documented. 


\section{Gradient-Driven Diffusion of Multi-Atom Molecules Through Macromolecules and Membranes: LDRD 96- 0021 Close-out Report}

\section{Introduction}

The goals of this Laboratory Directed Research \& Development (LDRD) effort were threefold: 1) to develop a new molecular simulation method able to model diffusion of multiatom molecules through macromolecules under conditions of a chemical potential gradient, 2) to develop a companion parallel algorithm to breach the computational challenges of the most important applications of the new method, and 3) to apply the new method to such problems. At the start of the project, no such method existed, thus the many important industrial and technological materials problems where gradient-driven diffusion of multiatom molecules is the predominant phenomenon were beyond the reach of molecular simulation. Three examples of such problems include: 1) diffusion in polymers, a fundamental problem underlying polymer degradation in aging weapons, 2) designing chiral polymer membranes in order to separate racemic mixtures into chirally pure compounds, and 3) the permeation of drug analogs through lipid bilayers to enable molecular engineering of drug delivery systems.

With the invention of dual control volume grand canonical molecular dynamics (DCVGCMD) [1], direct simulation of these gradient-driven diffusion processes became possible. This method yields not only molecular insight into the gradient-driven diffusion process but also provides a way to directly measure the diffusivity in the presence of chemical potential gradients. However, for the reasons outlined below, the original DCVGCMD method is restricted to gradient-driven diffusion of small molecules. Given that most of the truly important applications of such a molecular simulation method are for bonded species, (e.g. plasticizers in polymers, organics present in hazardous wastes, and pharmaceutical molecules) a new method was needed, one capable of generating chemical potential gradients in multi-atom species.

Finally, because calculating the flux is essential to using the DCV-GCMD method to obtain the diffusivity in the presence of a chemical potential gradient, systems with large crosssectional areas are necessary to obtain good statistics. Thus, a parallel algorithm was developed for the original DCV-GCMD method and embodied in a FORTRAN code called LADERA [2]. Extension of this parallel algorithm to the new DCV-GCMD method to enable parallel simulations of gradient-driven diffusion of multi-atom molecules was therefore another objective of this work.

The original implementation of the DCV-GCMD method in the code named LADERA treated only single-site (uniatomic) species. In this work, the method was extended first to enable the simulation of single-site species diffusing through a system comprised of multi- 
site (and single-site) species. Next, the method was generalized to allow the treatment of small multi-site species diffusing through multi-site species. Finally, a new implementation of the method, again in LADERA, was devised to enable the simulation of large, multi-site species diffusing through a multi-site system. Thus, in the text which follows, we begin with an overview of the DCV-GCMD method for simulating diffusion in systems with chemical potential gradients. This is followed by a brief description of the parallel algorithm developed previously for the single-site implementation of the method and some examples of applications of the original method. Next, the generalization of this parallel algorithm to enable the simulation of single-site species through multi-site systems (e.g. uniatomic gases through macromolecules) is presented and discussed followed by a prototype application of this capability. The extension of the DCV-GCMD method to diffusion of multi-site species through multi-site systems (e.g. polyatomic gas through macromolecules) is then discussed. The new method to enable the simulation of the gradient-driven diffusion of polyatomic species without employing insertions or deletions of the diffusing species is then presented as well as an exhaustive analysis of the intricacies of calculating diffusivities with this extended DCV-GCMD method.

\section{Dual Control Volume Grand Canonical Molecular Dynamics (DCV- GCMD)}

The dual control volume grand canonical molecular dynamics (DCV-GCMD) method developed by Heffelfinger and van Swol [1] and the similar method developed simultaneously by MacElroy [3] were designed to enable the simulation of diffusion and flow in systems experiencing chemical potential gradients. The basis for DCV-GCMD is the idea that if two local "grand canonical Monte Carlo control volumes" are placed inside the simulation volume of a molecular dynamics simulation, and grand canonical Monte Carlo (GCMC) insertions and deletions are carried out to establish different desired chemical potentials in these control volumes, equilibration exchanges between the MD simulation volume and the GCMC control volumes will establish a steady-state chemical potential gradient between the control volumes.

Because local control of the chemical potential underlies DCV-GCMD's ability to model systems with chemical potential gradients, DCV-GCMD differs from other methods of obtaining molecular dynamics simulations in the grand canonical ensemble (henceforth referred to as grand canonical molecular dynamics or GCMD methods). Such methods achieve a molecular dynamics simulation in the grand canonical ensemble without local control of the chemical potential and thus are inadequate for extension to systems with chemical potential gradients. An overview of these methods is provided elsewhere [4].

Briefly, DCV-GCMD can be thought of as a hybridization of $M D$ and GCMC because it simply consists of embedding two GCMC control volumes in a standard NVT molecular dynamics simulation (Figure 1). Each atom in the system is moved with the normal MD algorithm during an "MD phase". During this phase, the equations of motion are solved using the Verlet leap-frog algorithm [5]. Temperature control is achieved using either the constraint algorithm of Brown and Clarke [6] or the Nosé-Hoover extended system method 
[7]. After each $M D$ phase (which proceeds for a preset number timesteps), a "GCMC phase" follows.

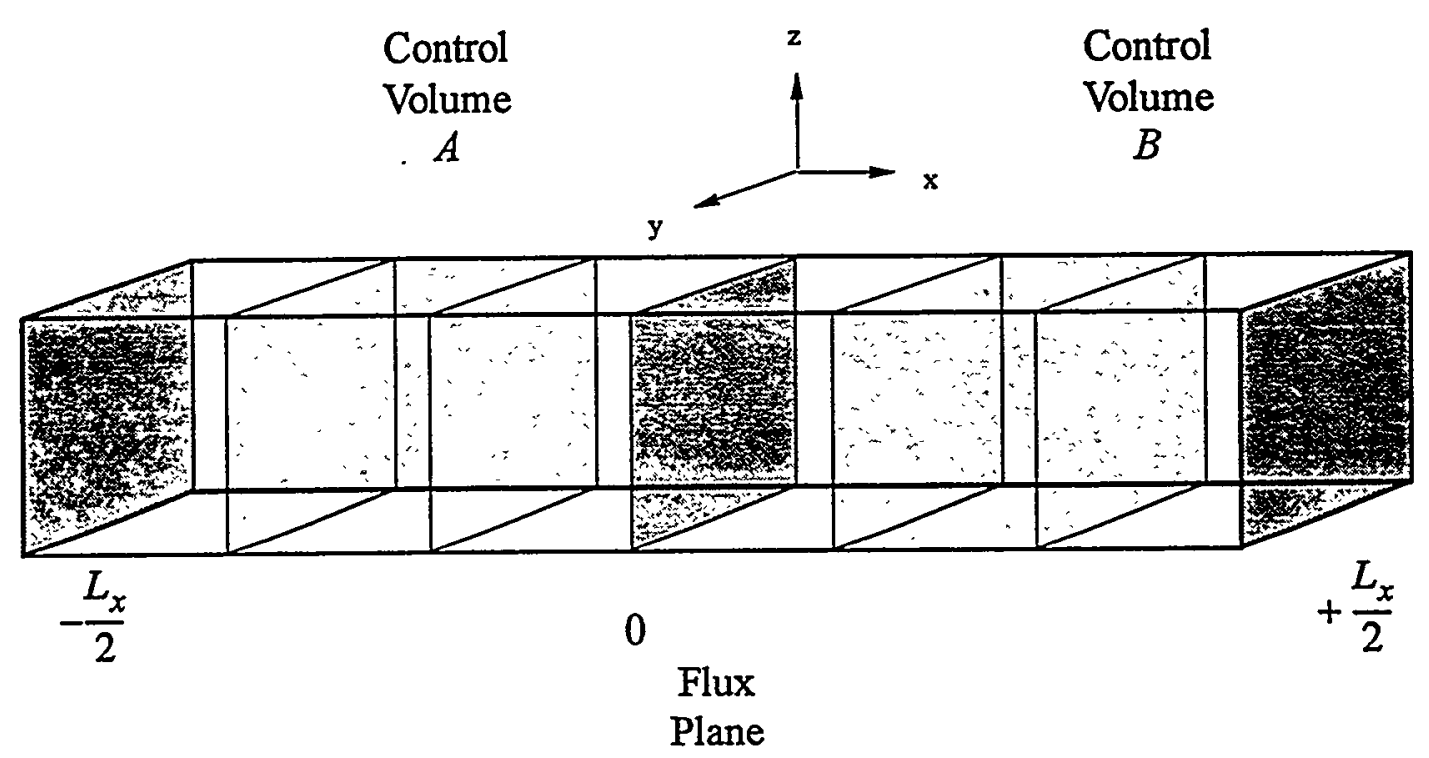

Figure 1. A schematic of a DCV-GCMD simulation. Two control volumes, $A$ and $B$, are used for insertions/deletions to achieve spatial chemical potential control of each species in the system. The flux planes are shown as the dark shaded planes between the control volumes and at the $x$-direction periodic boundaries, $x= \pm L_{x} / 2$.

During the GCMC phase, insertions, deletions, and (if desired) identity swaps of each component are attempted in each GCMC control volume in 8 cycles for reasons discussed below. Destroyed atoms are simply removed from the simulation while created atoms are assigned velocities chosen on a Gaussian distribution. After the GCMC phase, the simulation proceeds with another MD timestep, and so on.

During each of the $8 \mathrm{GCMC}$ cycles, a creation or destruction (chosen with equal probability) is carried out on species $i$ (chosen randomly). If the identity swap functionality is enabled [2], this is followed by an attempted change in type for a randomly selected atom of type $i$. For multicomponent mixtures, identity swaps greatly decrease equilibration time and enhance the ability to maintain constant chemical potential in the control volumes. The acceptance criteria [1] used for attempted insertions, deletions, and identity swaps are simply those of the standard GCMC method with the system volume, $V$, number of atoms of type $i$ in the system, $N_{i}$, and the desired chemical potential of type $i$ for the system, $\mu_{i}$, replaced with the volume of control volume $c, V(c)$, the number of atoms of type $i$ in control volume $c, N_{i}(c)$, and the desired chemical potential of type $i$ for control volume $c, \mu_{\mathrm{i}}(c)$, respectively. 
Periodic boundary conditions are applied in the dimensions appropriate for the simulated system: the $x, y$, and $z$ directions for simulations of diffusion in bulk systems and through amorphous porous materials; for systems confined in a pore, the pore is situated along the $x$-axis, thus periodic boundary conditions are applied only in the $x$-direction.

The density profile, $\rho_{\mathrm{i}}(x)$, is calculated by dividing the system volume into bins along the $x$-axis and averaging the number of atoms of type $i$ in each bin. The chemical potential profile, $\mu_{\mathrm{i}}(x)$, is calculated using the same bins as the density profile. Outside the control volumes, the chemical potential is determined via the insertion of test atoms whereas inside the chemical potential control volumes the calculated energies for attempted insertions are accumulated [2] and used to determine the chemical potential profile in the control volumes.

The flux between the two control volumes can be measured in three different ways. In the planar flux method (Figure 1), the net movement of each species type, $i$, across a stationary plane is accumulated as the simulation progresses and used to calculate the flux. In the control volume method, the net number of particles added or subtracted (total insertions total deletions) in control volumes $A$ and $B$ are accumulated and used to determine the flux for each type. In a third method, flux profiles are calculated using a binning system analogous to that used for the density profiles. For each bin, the sum of the $x$-velocities of the atoms of type $i$ is averaged over the simulation. This allows the flux of component $i$ to be calculated as a function of $x$-position. It should be noted that in all these cases, the fluxes are defined relative to the stationary reference frame of the simulation box.

\section{Parallel DCV-GCMD for Single-Site Species Diffusing In Single-Site Systems}

Because DCV-GCMD is a superposition of MD and GCMC phases, it can be parallelized in a straightforward fashion by simply applying the previously developed parallel algorithms for $\mathrm{MD}$ and $\mathrm{GCMC}$ to the $\mathrm{MD}$ and GCMC phases, respectively. For reasons discussed elsewhere [2], the MD and GCMC phases in the original LADERA code were parallelized with the spatial MD [8] and spatial GCMC [9] algorithms.

The parallel spatial $\mathrm{MD}$ algorithm consists of dividing the system volume into domains, one per processor. Each processor calculates the forces on the atoms in its domain, as well as their new positions. Before doing so, it must obtain the current positions of all atoms owned by surrounding processors which are within the force cut-off distance. This communication is conducted in an efficient iterative manner [8] which allows for force cutoffs which extend over an arbitrary number of processors. Obviously, both computation and communication increase as the force cut-off is increased. Next, each processor determines which, if any, atoms have left its domain. Departed atoms are removed from the coordinate arrays and placed in a communication array. This is then exchanged with neighboring processors, enabling each processor to pick up the atoms that have moved into its domain.

The parallel spatial GCMC algorithm employed during the GCMC phase involves subdividing each domain (the same domain as employed during the $M D$ phase) into 8 
subdomains. The GCMC-like insertions, destruction, and swaps, are carried out at the subdomain level on "control volume processors" (those responsible for domains lying in either control volume) while "profile processors" (those responsible for domains lying outside both control volumes) calculate the chemical potential profile, $\mu_{\mathrm{i}}(x)$, via test atom insertions. In both cases, the ideal gas contribution to the chemical potential is based on the volume of the subdomain and the number of atoms of each species in the subdomain. The energy calculation for proposed insertions, deletions, and identity swaps as well as for test atom insertions is carried out through a complicated interprocessor communication and calculation process which lies at the heart of the spatial GCMC algorithm $[2,9]$.

The parallel DCV-GCMD algorithm for single-site species diffusing in single-site systems was developed prior to this work [2]. While a primary goal of this work was to extend the DCV-GCMD method and LADERA code to enable the simulation of the gradient-driven diffusion in multi-site systems, a secondary goal was to develop a companion parallel algorithm for these extensions as well. The parallel algorithm for the single-site implementation, discussed above, was the basis for the new parallel algorithm developed for the extended method. This new parallel algorithm is discussed below.

\section{Examples for Single-Site Species Diffusing In Single-Site Systems}

Single-site GCMD methods which employ two local chemical potential control volumes (i.e. "dual control volume" GCMD) have been applied to bulk diffusion $[1,4,10]$, diffusion and flow in confined fluids [3,13-19], and diffusion of gases in polymers $[11,20,21]$. While the use of two local chemical potential control volumes as in DCV-GCMD is the only method available to obtain a dynamic simulation with a chemical potential gradient set a priori, other methods have been developed to accomplish similar objectives: primarily boundary condition methods where the atoms in a system change "color" or identity upon collision with a wall [22] or movement through a periodic boundary condition [23, 24] or the use of semi-permeable "walls" or boundary conditions [25], or simply maintaining a desired density at various places in the simulation volume [26-30].

The DCV-GCMD method has been applied to a variety of systems with the Lennard-Jones model for site-site interactions,

$$
\phi_{i j}(r)=\left\{\begin{array}{cc}
\phi_{i j}^{L J}(r)-\phi_{i j}^{L J}\left(r_{i j}^{c}\right) ; & r \leq r_{i j}^{c} \\
0 ; & r>r_{i j}^{c}
\end{array},\right.
$$

where $\phi_{i j}^{L J}(r)$ is the full Lennard-Jones potential,

$$
\phi_{i j}^{L J}(r)=4 \varepsilon_{i j}\left[\left(\frac{\sigma_{i j}}{r}\right)^{12}-\left(\frac{\sigma_{i j}}{r}\right)^{6}\right]
$$


Gradient-Driven Diffusion of Multi-Atom Molecules Through Macromolecules and Membranes: LDRD 96-0021 Close-out Report

and $r_{i j}^{c}$ denotes the cutoff distance of the ij-interaction. The mixing rules,

$$
\begin{aligned}
\sigma_{i j} & =(1-\alpha) \sigma_{i}+\alpha \sigma_{j} ; \quad i<j \\
\sigma_{j i} & =\sigma_{i j} \\
\varepsilon_{i j} & =\sqrt{\varepsilon_{i} \varepsilon_{j}}
\end{aligned},
$$

where $\alpha$ is an adjustable parameter which affects solution nonideality, are generally used to determine the cross-parameters.

For the purposes of the following discussion, the input chemical potential parameter employed by the LADERA code, $\mu^{\prime}$, is defined by

$$
\beta \mu_{i}^{\prime} \equiv\left(\beta \mu_{i}+3 \ln \left(\frac{\sigma_{i}}{\Lambda_{i}}\right)\right)
$$

\section{Color Diffusion}

The original work on the DCV-GCMD method [1] was designed to check the validity of the method for determining transport diffusivities in the presence of a chemical potential gradient. In order to enable comparison to equilibrium diffusivities obtained from a standard NVT molecular dynamics simulation, a color diffusion "experiment" was designed using the soft repulsive potential of Weeks-Chandler-Andersen $\left(r_{i j}^{c}\right.$ from eqn 1 is simply set equal to $2^{1 / 6} \sigma_{i j}$ ). Two components, with identical interaction parameters, were simply "labeled" as species 1 and species 2 . That is, the Lennard-Jones interaction parameters were taken to be,

$$
\begin{gathered}
\sigma_{11}=\sigma_{22}=\sigma_{12} \equiv \sigma, \text { and } \\
\varepsilon_{11}=\varepsilon_{22}=\varepsilon_{12} \equiv \varepsilon .
\end{gathered}
$$

Equal and opposite chemical potential gradients were established and the fluxes and steady state density profiles were used to calculate the transport diffusivities of each in the presence of a chemical potential gradient of that species. However, for the system as a whole (labeling aside), no chemical potential gradient existed. The transport diffusivities calculated from the fluxes and density profiles were found to match the self-diffusivity obtained from a standard NVTMD simulation at the same density [1].

\section{$\underline{\text { Uphill Diffusion }}$}

The DCV-GCMD method has also been used to investigate "uphill diffusion" in a bulk ternary WCA Lennard-Jones system $[11,10]$. In order to create nonideal conditions 
sufficient to establish the steady-state uphill diffusion phenomena; the interaction parameters were first varied. Ultimately, the $\alpha$ parameter in eqn 3 was taken to be equal to 0 , establishing sufficient nonideality to induce the uphill diffusion effect. The other interaction parameters for this system are listed in Table 1 . The temperature was taken to be $k T / \varepsilon_{1}=1.0$.

Table 1. Uphill Diffusion Lennard-Jones Parameters and Diffusivities

\begin{tabular}{|c|c|c|c|c|c|c|}
\hline \multirow{2}{*}{ Component } & \multicolumn{3}{|c|}{ Model Parameters } & \multicolumn{2}{c|}{$\begin{array}{c}\text { Chemical Potential* in } \\
\text { Control Volumes A and B }\end{array}$} & $\begin{array}{c}\text { Calculated } \\
\text { Fluxes }^{\dagger}\end{array}$ \\
\cline { 2 - 7 } & $\frac{\varepsilon_{i}}{\varepsilon_{1}}$ & $\frac{\sigma_{i}}{\sigma_{1}}$ & $\frac{m_{i}}{m_{1}}$ & $\left.\frac{\mu_{i}^{\prime}}{\varepsilon_{1}}\right|_{A}$ & $\left.\frac{\mu_{i}^{\prime}}{\varepsilon_{1}}\right|_{B}$ & $J_{i}^{x} \sigma_{1}^{3} \sqrt{\frac{m_{1}}{\varepsilon_{1}}}$ \\
\hline 1 & 1.0 & 1.0 & 1.0 & -0.28377 & -999.99 & 0.0011 \\
\hline 2 & 1.0 & 1.35 & 2.0 & 1.84714 & 2.46892 & -0.0001 \\
\hline 3 & 1.0 & 1.20 & 1.4 & -999.99 & 1.34956 & -0.0004 \\
\hline
\end{tabular}

*defined in eqn 4.

$\dagger$ The fluxes are defined relative to the stationary reference frame of the simulation box and a positive flux is defined as in the direction from control volume $A$ to control volume $B$.

The density and chemical potential profiles for this interesting system are contained in Figure 2 and Figure 3, respectively. These profiles were equilibrated for 4 million MD timesteps from a starting point of one atom per subdomain and averaged for 3.25 million MD timesteps. (There were 3072 grand canonical moves, insertions/deletions, attempted per MD timestep.) The total number of atoms in the system was approximately 15,000 . From the calculated fluxes (Table 1) we can see that component 2 is indeed diffusing up a steady-state concentration gradient.

\section{Parallel DCV-GCMD for Single-Site Species in Multi-Site Systems}

Because a major goal of this work was to extend the parallel DCV-GCMD code, LADERA, to accommodate multi-site systems, major changes made to both the MD and GCMC phases of LADERA [11].

In order to leverage data structures existing in the parallel multi-site MD code LAMMPS $[8,12]$, the MD phase of the original LADERA was replaced completely with much of the LAMMPS code. This provided such necessities as the intramolecular potential energy functions for the proper modeling of materials such as polymers and biomolecules, neighbor lists for its calculation of nonbond (van der Waals) pairwise forces, and several different options for maintaining constant temperature, calculating long-ranged (Coulombic) forces, and speeding integration [8]. Although not all of these additional 
Gradient-Driven Diffusion of Multi-Atom Molecules Through Macromolecules and Membranes: LDRD 96-0021 Close-out Report

options are currently utilized in LADERA, they may be implemented in the future as the need arises.

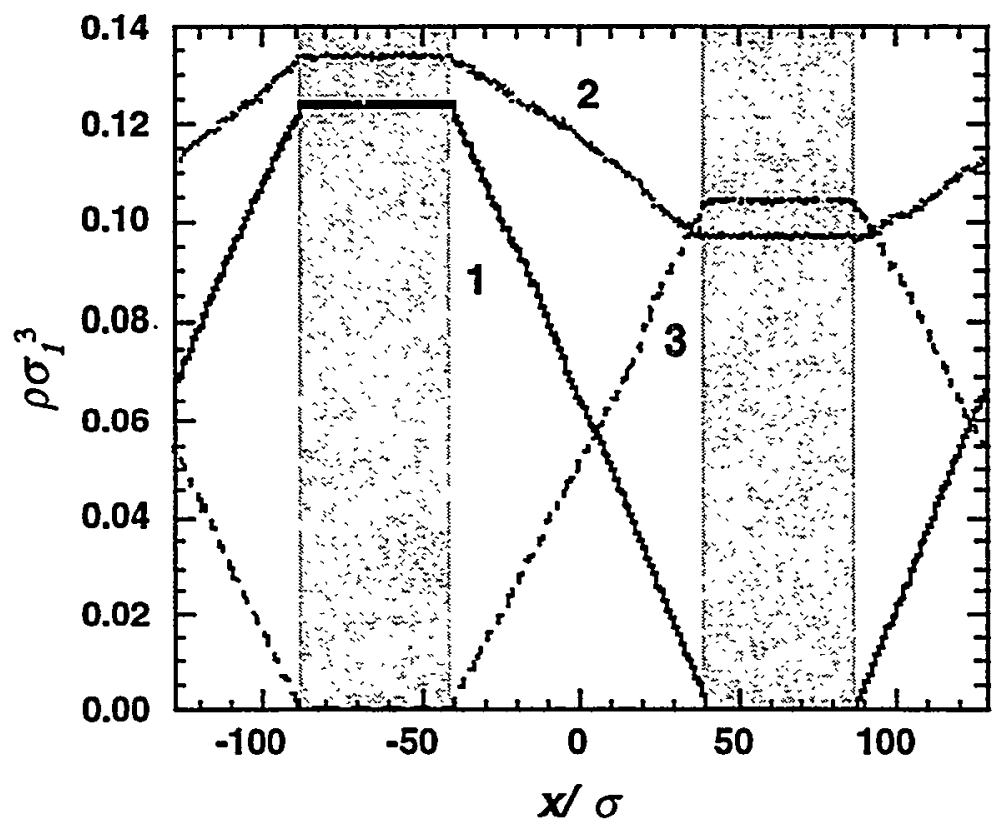

Figure 2. Density profiles for a WCA bulk ternary experiencing uphill diffusion in component 2. Each curve is labeled with its species number. The shaded regions represent two control volumes $A$ (left) and $B$ (right).The Parallel "MD Phase"

The spatial decomposition for molecular dynamics, discussed briefly above, is the basis for the parallel LAMMPS code, like the original LADERA code. LAMMPS supports several varieties of long-ranged (Coulombic) and short-ranged (Lennard-Jones) nonbond pairwise interaction potentials. Most importantly for the detailed modeling of molecular systems, several varieties of the following types of intramolecular interactions are also supported: bond stretching, angle bending, dihedral torsion, and improper bending. In order to implement these intramolecular potentials in the spatial decomposition algorithm, LAMMPS assigns a unique integer tag to each atom at the beginning of the simulation; this global tag is carried by that atom for the duration of the simulation. Each atom also carries a list of all intramolecular interactions for which it participates and lists of the global tags of partners in those interactions. Thus it is straightforward to find an atom's partners in for a given intramolecular interaction, even if some of them are assigned to a different processor, by looking for the correct global tag.

The use of neighbor lists by LAMMPS for short-ranged nonbond interaction pairs is significant to the implementation of the GCMC phase and deserves some discussion. The idea of a neighbor-list approach [5] is to build a list of atom pairs which are close enough that they may interact energetically over the next several time steps. This is typically done by including pairs that are within $\left(r_{c}+r_{s}\right)$ of each other, where $r_{c}$ is the cutoff in the 
pairwise interaction potential and $r_{s}$ is a "skin" distance. Then, for the next several time steps, all relevant interaction pairs in the system may be found by simply sweeping through these lists. New lists must be built when enough time has elapsed so that a pair not appearing in the current lists is within interaction range. The use of neighbor lists can provide a significant savings of time over the unmodified $\mathrm{MD}$ algorithm, where the distances between all atom pairs in the system are calculated at every time step to find interacting pairs.

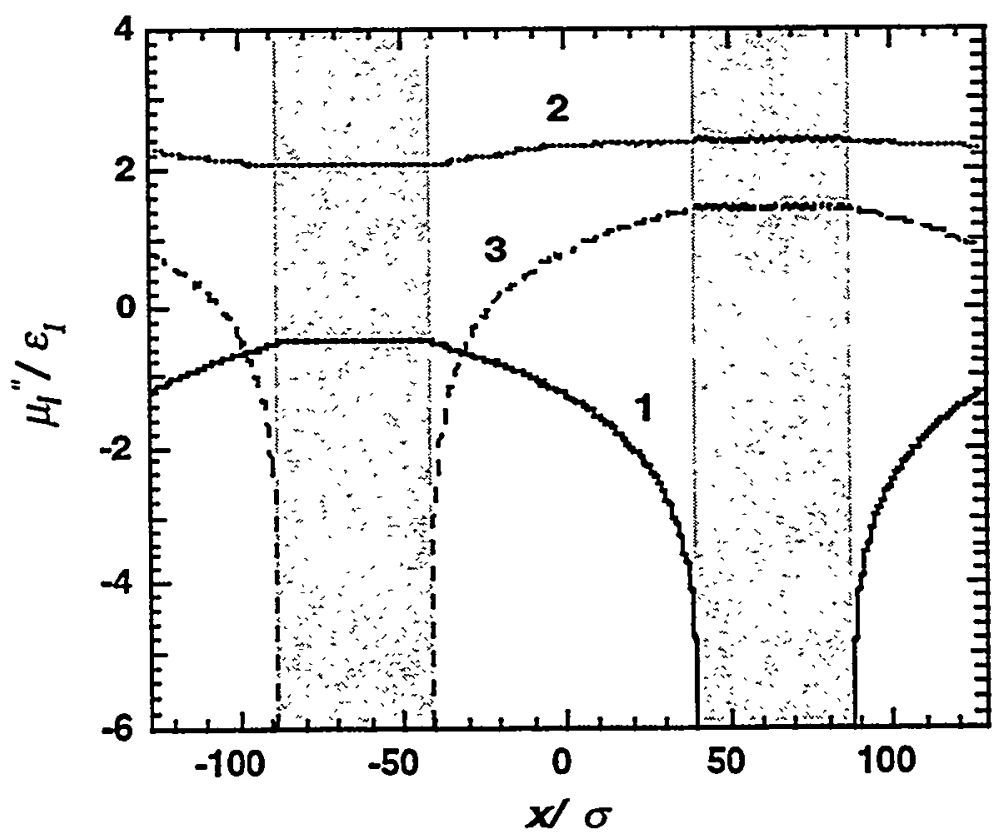

Figure 3. Chemical potential profiles for a WCA bulk ternary experiencing uphill diffusion. Each curve is labeled with its species number. The shaded regions represent two control volumes $\mathrm{A}$ (left) and B (right).

In the LAMMPS code, the building of new neighbor lists (a "re-neighboring") begins with each processor taking ownership of all atoms currently within its spatial domain. These will be referred to as atoms "owned" by the processor. The processor retains ownership of this set of atoms until the next re-neighboring, even if some move out of the domain during that time. A processor has complete information about each of its own atoms, including global tag, type (an integer label denoting the chemical species), position, velocity, number of intramolecular interactions in which it is participating, and partners in those interactions. In order to properly evaluate the forces on its own atoms over the next several timesteps, a processor must also know the global tag, type, and position of all atoms within $\left(r_{c}+r_{s}\right)$ of its borders. This information is obtained by a three-step communication process with all neighboring processors that might own such atoms; the details of this communication are described elsewhere $[2,8]$. At this point, each processor has two data structures: a set of owned atoms for which it has complete information and a set of nearby "borrowed" atoms for which it has only global tag, type and position information. From these data structures, 
each processor creates a list of all atom pairs with the following qualifications: the pair must contain at least one owned atom, and the atoms of the pair must be separated by a distance less than $\left(r_{c}+r_{s}\right)$. This list is referred to as the neighbor list, and a search of this list is sufficient to find all the pairwise nonbond forces acting on owned atoms. A pair which consists of two owned atoms is stored only once in the neighbor list, and Newton's 3rd Law is used to calculate the force on each atom. A pair which consists of one owned atom and one borrowed atom will also be found in the list of the processor which has ownership of the borrowed atom; each processor independently calculates the force on its own atom and Newton's 3rd Law is not used. There is an option in LAMMPS where such a pair is stored on only one of the processors and extra communication is done to pass the Newton's 3rd Law force, but it is not used with LADERA, for reasons that will be explained below. At this time, lists are also made of intramolecular interactions which contribute to the forces on owned atoms. This can be done in a straightforward way with the use of the global tags described above, as long as no two partners are more than $\left(r_{c}+\right.$ $r_{s}$ ) away.

LAMMPS uses the velocity Verlet algorithm [5] to integrate the equations of motion, rather than the leapfrog Verlet [5] of the original LADERA. A LAMMPS integration step proceeds with each processor first performing a full-timestep update of the positions and a half-timestep update of the velocities for its own atoms. These operations use the current forces on these atoms, which are calculated and stored at the end of the previous time step. At this point, LAMMPS determines if a re-neighboring is required. re-neighborings can be triggered at preset time intervals, or they can be delayed until an atom has moved a distance greater than $r_{s} / 2$ since the last re-neighboring; the latter option is often the more efficient. If a re-neighboring is required, then one occurs as described above. If one is not required, each processor needs an update of the positions of its borrowed atoms, which have just been moved by the processors which own them. This is done efficiently in LAMMPS by a swap of ordered lists with the same neighbors from which the borrowed atoms were obtained at the last re-neighboring. At this point, either by a rebuilding of the neighbor lists or by a swap, each processor knows the global tag, type, and current position of each atom in its lists. A force calculation is now done; each processor moves through its neighbor and intramolecular lists, calculating the force contribution of each entry and adding it to the force counter on the appropriate owned atom(s). The total force on each owned atom is then stored. The velocity updates are then completed, and thermodynamic calculations and temperature control are performed if required. The cycle is then repeated.

\section{The Parallel "GC Phase"}

The main ideas used in the GC phase of the parallel single-site version of LADERA [2] are retained in the parallel implementation of the parallel multi-site version. As before, the GC phase uses same domains that are used in the $\mathrm{MD}$ phase, with each of these domains divided into 8 subdomains to parallelize the GC moves. The domain size in LAMMPS is arbitrary, but when using it with LADERA we are restricted to subdomain edges which are greater than or equal to $r_{c}$ (and therefore domain edges which are greater than or equal to $2 r_{c}$ ) so that the independence of the parallel GC moves is maintained. At the beginning of 
the GC phase, each processor builds a list of atoms which are within its borders and indexes them by type and subdomain location. Each processor then cycles through its subdomains in an ordered progression, attempting insertions or deletions if it is a control volume processor or attempting "test" insertions if it is a "profile" processor [2]. The energy for these proposed changes is determined by the subdomain-based position/energy swap process $[2,9]$. Although these main concepts are the same, the implementation of the GC phase required some modification for compatibility with LAMMPS in the new parallel algorithm for multi-site LADERA.

The primary challenge in extending the LADERA parallel DCV-GCMD algorithm to macromolecular systems was making atom insertions and deletions compatible with the neighbor list format of LAMMPS. It is a fairly straightforward task for a processor which inserts an atom to find all pairs involving that atom and add these pairs to the neighbor list. The difficult issue is ensuring that the new atom is entered in the neighbor list of an adjacent processor, if necessary, as a borrowed atom. Deletions are accompanied by the same difficulty, plus an additional one: a pair of atoms which are owned by the same processor are stored only once in the neighbor list of the processor, so the deleted atom may appear in many (and widely scattered) places in the neighbor list.

To avoid these problems, we decided on the following approach to $\mathrm{GC}$ moves (insertions and deletions) in the new version of LADERA to be developed in this work. Instead of generating a random position only when an insertion is actually attempted, each processor generates an entire set of random positions just prior to the building of new neighbor lists. These random positions are assigned to ghost atoms, denoted as such by an assigned type of - 1 , which are placed in the "owned" data structure of each processor. There are $N_{\text {ghost }}$ of them in each subdomain, and their $(x, y, z)$ positions are distributed randomly in the space of that subdomain. Since these atoms are in the data structure and they have assigned positions, LAMMPS includes them in the building of neighbor lists, with the correct spatial neighbors, just as if they were "live" atoms. However, several lines of code were added to LAMMPS to bypass any force calculation involving a ghost atom and to bypass position updates for ghost atoms. Thus the ghosts are invisible to the dynamics but present in the data structure. They are used in the GC phase in the following way. When an insertion is attempted, the processor picks the next available ghost in that subdomain as the candidate. The energy of the insertion is evaluated based on the proposed insertion type and the position of the ghost. If the insertion is determined to be successful, the type of the ghost atom is changed from -1 to the insertion type, it is assigned a velocity chosen randomly from the Maxwell-Boltzmann distribution, and it is added to the subdomain list. It is now a real atom and will be considered when doing further insertions and deletions, force calculations, and position updates. If the insertion is not successful, the ghost atom is discarded as a candidate for future insertions and a pointer is set to the next one in line (using this ghost for future attempts would bias the Monte Carlo algorithm). A deletion is handled by simply turning the deleted atom to a ghost (changing its type to -1) and removing it from the subdomain lists. At the end of the GC phase, it is necessary to update all neighboring processors on the insertions and deletions just performed. This is done by simply swapping an ordered list of atom types with neighboring processors, as is done for 
Gradient-Driven Diffusion of Multi-Atom Molecules Through Macromolecules and Membranes: LDRD 96-0021 Close-out Report

the position swaps in LAMMPS. When the next re-neighboring point is reached, all ghosts are cleared from the data structures, and a fresh set for insertions is created.

There is an additional important point to note. If a control volume processor uses the final ghost in one of its subdomains, it no longer has the capability to insert an atom in that subdomain. Simply skipping such proposed insertions would lead to a bias in the Monte Carlo algorithm, so when a final ghost is used in any subdomain of a control volume processor, the processor sets a flag which will trigger a re-neighboring (and therefore a "reghosting") immediately following the GC phase. Profile processors are also assigned ghosts, which they employ in test insertions to calculate chemical potential profiles. Lack of ghosts in a profile processor does not trigger a re-neighboring; the proposed test insertion is simply skipped.

Another challenge was the generation of unique global atom tags for newly inserted atoms. The global tags, as discussed earlier, are used by LAMMPS to find intramolecular interaction partners. They are also required if Newton's 3rd Law is employed to calculate nonbond forces between atoms owned by different processors (see previous section). If these two features are to be used correctly, it is necessary for each atom to have a unique global tag. However, if we turn off the "inter-processor Newton's 3rd Law" feature, and we restrict ourselves to insertion and deletion of molecules comprised of only one atom (or "interaction site"), we can sidestep the problem by simply assigning a dummy tag to every inserted atom. We use a value of 0 for this dummy tag to avoid conflict with any valid tags (those having a value $>1$ ). There will eventually be many atoms in the simulation with a tag of 0 , but this will be of no consequence since these tags will not be used for any significant purpose. Multi-atom molecules with valid tags may still be present in the simulation, and their forces and position updates will be computed correctly, but they cannot be inserted or deleted. The inability to use the inter-processor Newton's 3rd Law feature does not generally result in a large computational loss. Plimpton [8] has pointed out that the savings gained by this feature scales as the number of "borrowed" atoms in the data structure and that it will diminish in importance as the domain size grows large relative to the range of the potential (as it will for very large problems). The more serious restriction of the dummy tag approach is the lack of ability to insert and delete multi-site species. This initially limited the new LADERA to the modeling of single-site species diffusing under a gradient through any type of bonded system. However, follow-on work provided a method which allowed the exchange of bonded species and is discussed below.

There is an additional, less obvious challenge in making LADERA compatible with LAMMPS. With to the original parallel GC algorithm, any molecule (of an insertable/ deletable type) which is within the boundaries of a subdomain must be considered as a candidate for deletion from that subdomain [2]. In LAMMPS, between re-neighborings, it is possible for atoms to leave the spatial domain of the processor which owns them; in fact, this is a common occurrence at liquid densities. Thus one may end up in the unpleasant situation where a processor has selected an atom for deletion which it does not own. Such an event is not thermodynamically incorrect, but it is difficult to handle computationally. This problem did not exist in the original LADERA code, because neighbor lists were not used and ownership of an atom was reassigned immediately upon the crossing of a domain boundary (the "MD-II communication" in [2]). For the present case, one can devise 
schemes where the processor would communicate with neighboring processors to find the owner of the molecule, which would then perform the deletion. However, such schemes generally require unique global atom tags, and more importantly, significant interprocessor communication. So we use a different approach in which a sub-subdomain is set up inside each subdomain [11], defined by boundaries which are parallel to those of the subdomain, but displaced inward by a distance $\Delta_{s u b}$. All insertions and deletions are now performed only within the sub-subdomains. It is still necessary to make a list of all atoms within the subdomain for the purpose of energy calculations but it is also necessary to have a list of the subset of these which are within the sub-subdomains. Candidates for deletion are chosen only from this subset list. Likewise, insertions are only done within the boundaries of the sub-subdomains. In the GC formulae for insertion and deletion [2], the volume used is now that of the sub-subdomain, and the $N_{i}$ are now the total number of molecules of type $i$ in the sub-subdomain. The purpose of this setup is to delay the entrance of molecules owned by other processors into the spatial region where insertions and deletions are performed, thus delaying the situation described above. If at any time a processor does choose an atom owned by another processor for deletion, the deletion is skipped and the processor sets a flag which will trigger a re-neighboring in LAMMPS immediately following the $G C$ phase. A larger $\Delta_{s u b}$ results in fewer of these forced re-neighborings. In fact, if $\Delta_{s u b}$ is set greater than $r_{s} / 2$, such occasions will be virtually nonexistent. The problem with a large $\Delta_{s u b}$ is the creation of "dead space" - regions in the control volumes where no insertions or deletions can be performed. This can lead to problems in maintaining the desired chemical potential and density values in the control volumes [11].

\section{The Resulting Parallel DCV-GCMD Algorithm}

The GC phase is driven by a subroutine which is hooked into the LAMMPS MD step after the first velocity update. The first step in the $G C$ phase is a swap, where processors exchange ordered lists of position information for borrowed atoms; this is necessary because atoms have just completed a move. Next, indexed lists of atoms are made for each subdomain and sub-subdomain. The sub-subdomains are then cycled through in a parallel fashion, with proposed insertions and deletions, energy calculations, and acceptance decisions. The ghost atom approach, as described above, is used here. Checks are made to see if any processor has run out of ghosts; if so, a flag is set to trigger a re-neighboring (and therefore a "re-ghosting") upon the return to LAMMPS. The selection of a non-owned atom as a deletion candidate will also trip a flag to force a re-neighboring. Upon the return to the LAMMPS code, the decision is made whether to re-neighbor. A re-neighboring is performed if it is required, and all lists are then current. If a re-neighboring is not required, it is necessary to perform a swap to determine the current types of borrowed atoms, because some of these may have changed in the GC phase just completed. At this point, whether by re-neighboring or swapping, each processor has enough information to calculate the forces on its atoms. Once this is done, the forces are stored, and the cycle is repeated. 
Gradient-Driven Diffusion of Multi-Atom Molecules Through Macromolecules and Membranes: LDRD 96-0021 Close-out Report

\section{Demonstration Calculations and Performance Characteristics}

Below we describe three model systems used to evaluate the performance of LADERA on two massively parallel platforms which the code was run. We demonstrate the increased speed of the modified LADERA as compared to the original LADERA for a singleinteraction-site fluid model. We also explore the optimization of the new parameters $r_{s}$, $\Delta_{\text {sub }}$ and $N_{\text {ghost }}$. Finally, scaling results are given for the new code on the two platforms.

\section{Model Systems}

Three model systems were employed to investigate the efficiency of the new algorithm. System $A$ is the same as described above (equations 1-6) for the original DCV-GCMD color diffusion work [1]. As before, both species are inserted and deleted, and the system is designed to be symmetric: in control volume $A$, the chemical potentials are set to $\mu_{1}{ }^{\prime} / \varepsilon=1.54$ and $\mu_{2}{ }^{\prime} / \varepsilon=2.34$, while in control volume $B$, they are set to $\mu_{1}^{\prime} / \varepsilon=2.34$ and $\mu_{2}{ }^{\prime} / \varepsilon=1.54$. The potential cutoff $r_{c}$ is set to $1.12246 \sigma$.

System B1 consists of the LJ species 1 and 2 of System A, plus a polymer component, species 3 . The polymer is a set of linear chains with 50 interaction sites (beads) each, where neighboring beads in a chain are linked by FENE bonds,

$$
U_{b o n d, i j}=-\frac{1}{2} k r_{o}^{2} \ln \left[1-\left(\frac{r_{i j}}{r_{o}}\right)^{2}\right]+4 \varepsilon\left[\left(\frac{\sigma}{r_{i j}}\right)^{12}-\left(\frac{\sigma}{r_{i j}}\right)^{6}\right]+\varepsilon
$$

where $k$ is a spring constant $r_{o}$ is the maximum bond length, and $\varepsilon$ and $\sigma$ are the usual $\mathrm{LJ}$ parameters. The first term in eqn 7 extends to $r_{o}$, and the second term is truncated at $r_{c}$. A polymer bead has $\mathrm{LJ}$ interactions with all species 1 and 2 molecules and all other beads which are not its nearest neighbors on the same chain (note that a $\mathrm{LJ}$ interaction between nearest neighbors is already included in the FENE bond). All beads and species 1 and 2 molecules have an identical mass $m$ and identical $L J$ parameters $\sigma$ and $\varepsilon$. In system $B 1$, only species 1 and 2 are inserted and deleted. Again, the system is designed to be symmetric; in control volume $A$, the chemical potentials are set to $\mu_{1}{ }^{\prime} / \varepsilon=2.13$ and $\mu_{2}{ }^{\prime} / \varepsilon=-999.0$, while in control volume $B$, they are set to $\mu_{1}{ }^{\prime} / \varepsilon=-999.0$ and $\mu_{2}{ }^{\prime} / \varepsilon=2.13$. The value of -999.0 is low enough to force the density essentially to zero. The polymer is present in the simulation box at a total bead density of $\rho \sigma^{3}=0.25$. These values produce a system which is approximately $50 \%$ polymer by weight. The cutoff $r_{c}$ for all interactions is again $1.12246 \sigma$. The parameters $k$ and $r_{o}$ were set to $30.0 \varepsilon / \sigma^{2}$ and $1.5 \sigma$, respectively.

System B2 consists of the same components as system B1, but in this system, the reduced bead density of the polymer is set to $\rho \sigma^{3}=0.85$. In control volume $A$, the chemical potentials are set to $\mu_{1}{ }^{\prime} / \varepsilon=5.0$ and $\mu_{2}{ }^{\prime} / \varepsilon=-999.0$, while in control volume $B$, they 
are set to $\mu_{1}^{\prime} / \varepsilon=-999.0$ and $\mu_{2}^{\prime} / \varepsilon=5.0$. These values produce a system which is approximately $99 \%$ polymer by weight. The cutoff $r_{c}$ for all interactions is again $1.12246 \sigma$.

All systems were simulated at a reduced temperature of $k_{B} T / \varepsilon=1.0$, where $k_{B}$ is Boltzmann's constant.

\section{Speedup on Single-Site Systems}

The simulations were performed on two multiple-instruction/multiple-data parallel machines at Sandia. One is a 1024-processor nCUBE2 with custom scalar chips capable of $\sim 2$ Mflops peak each, connected in a hypercube topology. The other is an 1824-processor Intel Paragon with i860XP processors connected in a two-dimensional mesh.

It is of interest to compare the speed of the new LADERA with that of the original LADERA. Of course, the original LADERA is not capable of simulating bonded systems, so we used the single-site system $A$ described above to compare the two. The simulations were performed in identical simulation boxes on 32 processors in an $8 \times 2 \times 2(x y z)$ array, on the nCUBE2. For the new LADERA run, the following values were used: $r_{s}=0.5 \sigma, \Delta_{\text {sub }}$ $=0.25, N_{\text {ghost }}=9$. These were not obtained from a rigorous optimization but were simply chosen as reasonable values based on experience. The MD timestep used in this study was $0.01\left(m \sigma^{2} / \varepsilon\right)^{1 / 2}$. Each run consisted of 20,000 steps to equilibrate the system from zero density, followed by 25,000 steps over which the CPU time was averaged. The original LADERA code used 1.80 CPU seconds per time step, while the new LADERA required just $0.51 \mathrm{CPU}$ seconds per time step. It is clear that even without fine-tuning of the new parameters, the new LADERA algorithm provides a significant speedup. This

improvement is of course due to the use of neighbor lists, which increase the efficiency of the force calculations. Timing breakdowns show that the CPU time spent by the original LADERA in determining forces exceeded that spent by the new LADERA by a factor of 7 (this comparison includes the neighbor-list building time in the new LADERA). Since the force calculation is roughly half of the total CPU time in the old LADERA, this accounts for the factor of 3.5 improvement in overall speed. Interestingly, this was achieved even though there were nearly as many ghosts in the data structure as real atoms for this particular system and value of $N_{\text {ghost }}$.

\section{Optimization of new parameters}

Due to the use of neighbor lists, the new LADERA algorithm includes three new parameters that the original LADERA did not: $r_{s}, \Delta_{\text {sub }}$, and $N_{\text {ghost }}$. In this section, we present guidelines for choosing optimal values of these parameters, which is a complex task because their effects are highly coupled.

Consider the choice of a $\left\{r_{s}, N_{\text {ghost }}\right\}$ pair. Some insight may be gained by first considering the problem of optimizing $N_{\text {ghost }}$ for a given value of $r_{s}$. Since a natural frequency for reneighborings in a system is set by the time required for an atom to travel a distance $r_{s} / 2$, it will certainly be unprofitable to use more ghosts than are required to span that time. The consequences of using fewer ghosts are not as obvious; fewer ghosts will require more 
reneighborings, but the reneighboring process will be faster since there will be fewer atoms in the data structure. In Figure 4 we have plotted CPU seconds per time step as a function of $N_{\text {ghost }}$, for system BI with $r_{s}=0.5 \sigma$ and $\Delta_{\text {sub }}=0.25 \sigma$. Plotted against the right abscissa are the total number of reneighborings and the number of reneighborings triggered by lack of ghosts. The number of reneighborings caused by non-owned atoms entering subsubdomains was zero in all cases, so the total number of reneighborings is the number triggered by lack of ghosts plus the number triggered by an atom traveling a distance $r_{s} / 2$. The CPU time curve shows a distinct minimum near an $N_{\text {ghost }}$ value of 8 or 9 . Interestingly, this minimum occurs at the same point where the two reneighboring curves begin to split. This characteristic was observed for several other systems as well. These results imply that for a given $r_{s}$, one should use just enough ghosts to make it to the next reneighboring triggered by atomic motion. The use of more ghosts results is a waste of time in building neighbor lists, as expected. Interestingly, any fewer is also less efficient. Apparently, in that regime, the incremental savings in list-building time associated with lowering $N_{\text {ghost }}$ by 1 is offset by the cost of having to reneighbor 1 step earlier. It is possible that deviations from this behavior might occur in very dilute systems, but we have not examined this.

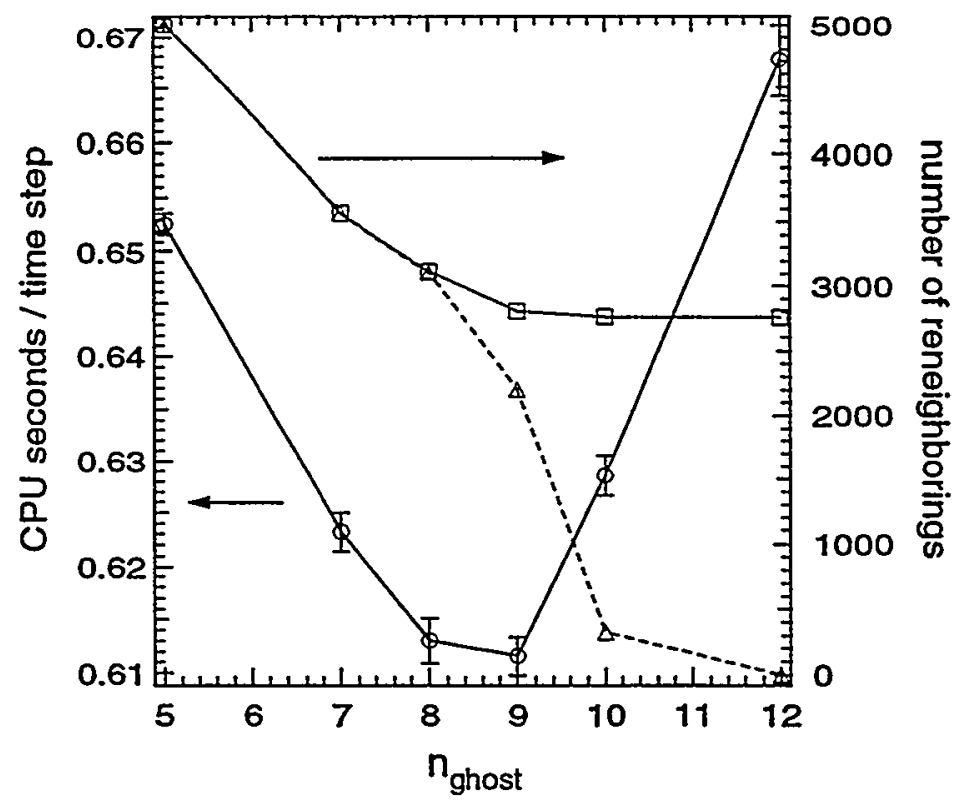

Figure 4. CPU seconds per time step and number of reneighborings as functions of $n g h o s t$, for system B1 with $r_{s}=0.5$ and $\Delta_{\text {sub }}=0.25$ on the nCUBE2. The circles represent the CPU time, plotted against the left abscissa; the error bars are the standard deviations, as determined from 5 averages over 5,000 time steps each. The squares represent the total number of reneighborings, plotted against the right abscissa. The triangles represent the number of reneighborings triggered by lack of ghosts, also plotted against the right abscissa. The various connecting lines are guides to the eye. 
Now that the behavior of $N_{\text {ghost }}$ is understood for a given $r_{s}$, the optimum value of the latter variable must be found. The best choice for $r_{s}$ has been discussed for normal MD simulations $[5,8]$. The basic idea is that a trade-off exists; increasing the skin size decreases the frequency of reneighborings, but it also increases the computation required in each force calculation and at each reneighboring (due to longer lists). The optimum value is generally found by trial and error on a case-by-case basis, because it depends on system size, density, temperature, and potential model. It is usually found to be about $20 \%$ of $r_{c}$ for liquid systems. We expect that the optimum value of $r_{s}$ for the new LADERA will also be in this neighborhood. However, because of the ghost atoms, this value will probably not correspond exactly to the optimum value for $r_{s}$ in a normal $\mathrm{MD}$ simulation on an identical system. Ghost atoms can be present in large numbers, even equal to the number of real atoms, and therefore they can significantly increase the work of building neighbor lists. This may skew the optimum $r_{s}$ in the new LADERA to a lower value than would be found in the corresponding MD simulation; however, we have not made a systematic comparison of $r_{s}$ values in the two types of simulation.

The $\Delta_{\text {sub }}$ parameter presents some additional complications. It is desirable to set this parameter to as small a value as possible in order to minimize the dead space in the control volumes. However, a value of $\Delta_{\text {sub }}$ less than $r_{s} / 2$ will cause forced reneighborings by the GC phase due to the presence of non-owned atoms in the sub-subdomains. Such reneighborings are very inefficient since they are triggered by the movement of an atom over a distance $\Delta_{\text {sub }}$, rather than the distance $r_{s} / 2$ on which the neighbor lists were based; the neighbor lists will therefore include many "wasted" pairs which never move closer than $r_{c}$ between the forced reneighborings. The best choice for a value of $\Delta_{\text {sub }}$ is therefore always $r_{s} / 2$. Fortunately, typical optimum values of $r_{s}$ seem to yield values of $\Delta_{\text {sub }}=r_{s} / 2$ which are acceptable in terms of dead space (see for example the system contained in Figure 6 below). However, there may be situations where the originally chosen optimum value of $r_{s}$ produces a $\Delta_{\text {sub }}=r_{s} / 2$ value which leads to unacceptable dead space effects. In such cases, it is necessary to reduce the value of $\Delta_{\text {sub }}$ until the density profiles are acceptable. The value of $r_{s}$ should also be reduced such that $r_{s}=2 \Delta_{\text {sub }}$ is maintained; this will assure that the best computational efficiency is achieved for the new value of $\Delta_{\text {sub }}$.

\section{Scaling with System Size}

The scaling performance with system size is a general concern in molecular simulations on parallel machines. We explored the scaling properties of the new LADERA code with system B1 on the nCUBE2 and system B2 on the Intel Paragon. Our approach was to hold the intensive properties of the system constant while adding on more spatial domains (and corresponding processors). Figure 5 is a plot of CPU seconds per time step as a function of the number of processors. We could have equivalently used as our ordinate the total simulation volume or the average total number of atoms, since these quantities are proportional to the number of processors. To provide an idea of the size of the simulations, the 1024-processor simulation on the Paragon contained 187,000 atoms on average, while the 64-processor simulation on the nCUBE2 contained about 7,000. The algorithm scales 
extremely well with system size, with only slight deviations from ideality at small system sizes on the nCUBE2. Although system B2 is at a higher density than system B1, the greater computation rate of the Intel processors results in a smaller overall CPU time. It should be mentioned that $r_{s}=2.0 \sigma$ was used in all of these simulations; this is probably far from the optimum value for these systems, so we expect that the CPU times could be unilaterally reduced by a factor of three or four for each system.

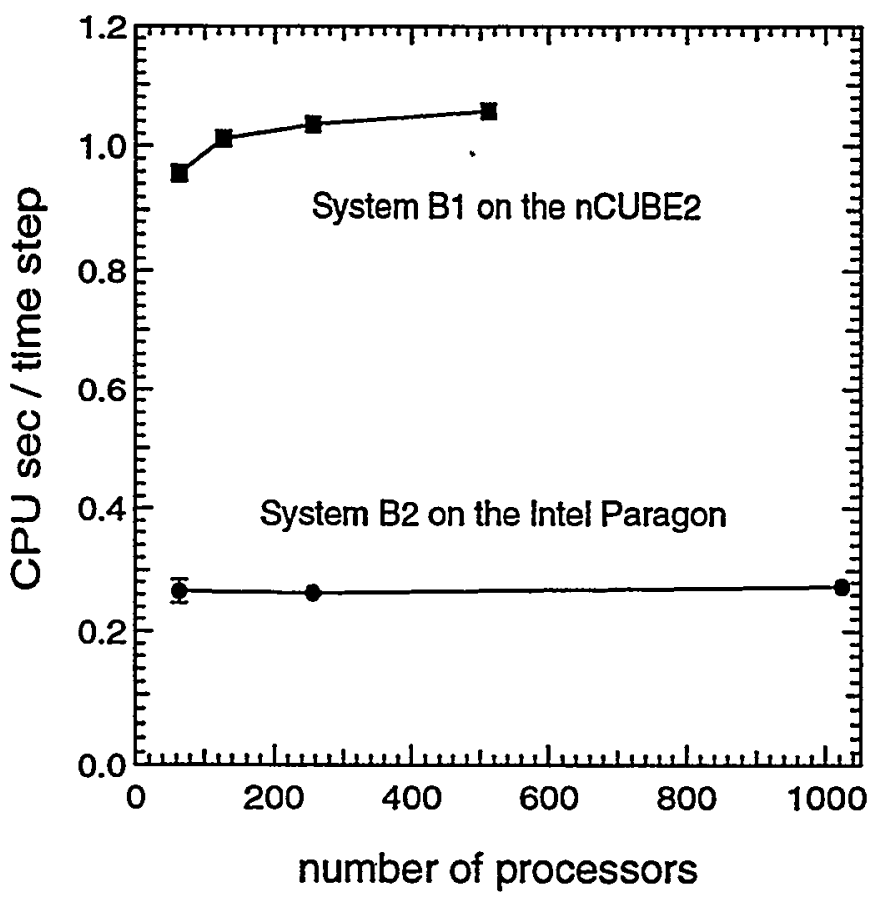

Figure 5. CPU seconds per time step as a function of number of processors (a measure of system size). The upper curve is for system B1 on the nCUBE2. The lower curve is for system B2 on the Intel Paragon. The value of $r_{s}$ is $2.0 \sigma$ for both curves.

\section{Parallel DCV-GCMD for Multi-Site Species in Multi-Site Systems}

Once a parallel multi-site system capability was implemented into LADERA, we began working on extending this capability to the gradient-driven diffusion of multi-site species in multi-site systems. The essential technical problem here is again best explained in terms of the two phases comprising the DCV-GCMD method, the MD and GC phases. While laborious, the previous work of extending DCV-GCMD to multi-site systems simply required enabling the single-site insertion/deletion in the $\mathrm{GC}$ phase to continue while generalizing the $\mathrm{MD}$ phase to bonded systems. As discussed above, this was accomplished by modifying the LADERA code to employ the LAMMPS code for its MD phase. The next step, enabling the DCV-GCMD method to model gradient-driven diffusion of multi-site species was carried out in two different ways. The first and most obvious approach is discussed here: developing a multi-site $\mathrm{GC}$ phase by performing insertions and deletions on multi-site species. The second, redesigning the chemical potential control method to 
enable control without insertions or deletions was also investigated and is discussed in the following section (Chemical Potential Control for Large Molecules).

Enabling insertion and deletion of multi-site species presented two primary challenges:

- Global Tags. As discussed above, after the implementation of parallel MD for bonded systems in LADERA, inserted atoms were assigned a global tag of zero. This was acceptable as global tags were only needed to compute forces due to bonded interactions. However, in order to insert molecules, a method is required to generate unique global tags for atoms being inserted on different processors.

- Ghost Atoms. Similarly, LADERA's algorithm at this point required atoms which were inserted and deleted via changing a ghost atom into a real atom or vice-versa. This enabled insertions and deletions without having to update the neighbor lists used in force calculations. While this idea could have be extended to handle polyatomic insertions and deletions, the additional overhead introduced by the ghost particles would be significant, even for atomic systems. Polyatomic systems would require a great many more ghost particles, for two reasons. Firstly, a separate set of ghost molecules would be required for each exchanged species, whereas previously, ghost particles could be used interchangeably by all exchanged species. Secondly, the acceptance rates for multi-site atoms decrease exponentially with the number of sites meaning that the required number of attempted insertions is much greater than for atomic species. Hence, a method was required for performing exchange moves without using ghosts atoms.

The solution to these problems is molecular insertion and deletion: insertion and removal of entire molecules, as opposed to just atoms. However, this approach requires a significant amount of additional information. In order to avoid constructing many new data structures, which would have to be maintained and communicated between processors, we tried to work within the existing framework of LADERA. Several major modifications to the code were made to enable multi-site insertions and deletions, ultimately producing a version of LADERA capable of simulating the diffusion of small, multi-site species in multi-site systems:

\section{Dynamic Tag Reallocation}

This procedure was developed to ensure that newly inserted atoms were assigned to the lowest available unused tag, which minimized the size of the global tag array. This is important for large systems, as the global tag array is the only data-structure in the code which scales as the total system size. The dynamic reallocation scheme introduced a certain amount of communication, which was necessary to ensure that atoms on different processors were not assigned the same tag. The procedure used is as follows. During each GC cycle, each processor maintains two lists, a free-tag list containing the $N_{\text {free }}$ global tags of atoms which have been deleted, and a need-tag list containing the $N_{\text {need }}$ atoms which have just been inserted (and do not yet have tags). At the end of the cycle, a call is made to a new routine called GLOBAL_TAGS. In this routine, the individual free-tag lists are merged across all processors. The merged free-tag list may also include some left-over 
Gradient-Driven Diffusion of Multi-Atom Molecules Through Macromolecules and Membranes: LDRD 96-0021 Close-out Report

free-tags from the previous cycle. The processors then stand in line to take tags from the free-tag list for their $N_{\text {need }}$ atoms. By performing a collective scanning sum on $N_{\text {need }}$, processors can figure out how many tags are needed by all the processors ahead of it in the line. In this way, each processor can pick out tags for its atoms from the free-tag list in such a way that no free-tag is skipped and no free-tag is picked twice. If there are more than enough free-tags, then the lowest tags are used and remaining free-tags are saved until the next cycle. Conversely, if there are not enough free-tags to go around, all of them are used, and additional tags are allocated from the end of the list.

\section{Elimination of Ghost Particles}

The ghost particle work-around was eliminated by forcing LAMMPS to recompile the neighbor lists after every $\mathrm{GC}$ phase. This simply means that the forces calculated after each GC phase would be calculated using an up-to-date neighbor list. With the previous LADERA scheme, this approach would result in LAMMPS performing a time-consuming re-neighboring process every timestep which would defeat the purpose of using neighbor lists. This situation was avoided by only performing a GC phase every $N_{\text {exchange }}$ timesteps, where $N_{\text {exchange }}$ is a new input variable. To compensate for the reduced frequency of the GC exchange phase, an increased number of GC cycles were performed during each $\mathrm{GC}$ phase, enumerated by another new input parameter, $N_{\text {cycle }}$. Provided that $N_{\text {exchange }}$ is not unrealistically large, which would introduce periodic behavior, the reduced frequency of the GC phase can be fully compensated by increasing $N_{\text {cycle }}$. In this way, the overall rate of insertions and deletions can be set at an arbitrary level while maintaining the reneighboring frequency at a level consistent with the LAMMPS skin-distance $r_{s}$ criterion (see previous section on single-site species in multi-site systems).

\section{Communication-free Energy Calculation}

The method for calculating the energy was changed in order to make it more efficient by exploiting several existing LAMMPS data structures. The reason for doing this was that existing LAMMPS data structures include a list of all atoms on the current processor as well as all atoms on other processors within the force cut-off. Hence, energies can be calculated without doing any additional interprocessor communication, obviating the need for the parallel Monte Carlo scheme of Heffelfinger and Lewitt [2, 9]. This resulted in a great reduction in the overall time required for energy calculation but introduced an additional problem. In the original LADERA code, insertions and deletions of atoms were not communicated to other processors until the end of the GC phase. This was acceptable as the energy calculation was based on atoms residing in the local processor domain. However, because the new scheme requires processors to know about certain atoms in neighboring processor domains, successful insertions and deletions must be communicated to neighboring processors before attempting the next insertion or deletion. One very inefficient way to do this would be to call the LAMMPS routines BORDERS and EXCHANGE after each attempted insertion or deletion. These routines completely reconstruct the list of atoms in each processor's domain and in neighboring domains within 
the force cut-off and are therefore very time consuming. However, testing of this approach demonstrated that it was about as fast as the previous approach. Ultimately the calls to BORDERS and EXCHANGE were replaced by a call to a new streamlined subroutine GC_BORDER which communicates only information on atoms which have just been inserted and deleted. Since inserted atoms have not yet been assigned global tags at this point, another call must be made to a routine called GC_TAG_EXCHANGE after the call to GLOBAL_TAGS. This routine communicates the tags of newly-inserted atoms to neighboring processors.

\section{$\underline{\text { Molecular Insertion and Deletion }}$}

Ultimately, the ability to insert and delete multi-site species enabled the extension of the LADERA code to model gradient-driven diffusion of small multi-site molecules. Unsurprisingly, however, several implementation challenges had to be overcome.

To begin, data structures needed to be established for the exchange species (those to be inserted or deleted). This was accomplished by establishing a set of templates for the molecular architecture of the exchange molecules. Each template specifies the molecule style (atomic, linear or non-linear) and the number of atoms in the molecule. Then, for each atom the following information is provided: type, charge, relative position, and details of all the bonded structures (up to third-nearest neighbor atoms) in which the atom participates. The relative positions are defined with respect to the centroid of the molecule, which would be its center of mass if all atoms in the molecule were identical.

Next, a multi-site insertion algorithm was developed which begins by first establishing the position of the centroid for a proposed molecule insertion and randomly picking a point in the sub-subdomain volume. Molecule orientation is next established by randomly selecting zero, two or three Euler angles, depending on the molecule style. Each atom of the molecule is then inserted, in turn, at a position calculated from the reference point, the relative coordinates and the rotation matrix corresponding to the Euler angles. The interaction of each atom with the system is calculated as described above. If any atom overlaps strongly with another atom in the system, the insertion attempt is immediately rejected. If all the atoms in the molecule are inserted without overlap, then the Boltzmann factor for the total interaction energy is calculated.

Acceptance or rejection of a proposed molecular insertion is determined using the same GCMC acceptance criterion as was used for atomic systems with some subtle distinctions. The density, chemical potential and energy are defined on a per-molecule basis. In addition, the above procedure disregards the effects of internal degrees of freedom, thus the molecules are inserted in a fixed conformation, which should be chosen to be close to the average value. The assumption made here is that the partition functions of the rotational and internal degrees of freedom are identical to those of an isolated molecule. This is a very good approximation for stiff molecules, such as oxygen, nitrogen and water, but breaks down for molecules with flexible internal modes, such as butane. If need arises in the future, a fourth molecule type can be added which will require a more detailed description and will randomly sample internal degrees of freedom as part of the insertion process. This 
capability may also arise out of work being planned at the University of Minnesota, which would introduce configurational bias Monte Carlo insertions into the code.

In order to attempt to delete a molecule from a subdomain, it is first necessary to maintain a list of the first atoms (called key atoms) of each molecule of each exchange type whose centroid lies in the sub-subdomain volume. A deletion attempt is performed by randomly selecting one of these atoms. The remaining atoms in the molecule can be identified using an array named SPECBOND, which is maintained by LAMMPS and keeps track of all atoms which are first, second or third neighbors. This is sufficient to handle molecules as complex as $n$-pentane (the key atom must be the third carbon), but not $n$-hexane. It is unlikely that exchange of such a long molecule would be attempted with the existing code, but if necessary, this could be achieved either by adding dummy bonds, by augmenting the SPECBOND array to include atoms beyond third neighbors, or by using an recursive method to step through the molecule.

Using the above procedure, the interaction energy of each atom with the rest of the system is calculated, taking care not to inadvertently calculate any intramolecular interactions. Then the Boltzmann factor for the total interaction energy of the molecule is calculated. Acceptance or rejection of the deletion is determined using the same GCMC acceptance criterion as was used for atomic systems, with the same caveats as were noted in the case of insertion.

The observant reader will have noted that molecular deletion may result in removal of atoms which do not lie within the sub-subdomain, even though the centroid does. Similarly, molecular insertions may result in the creation of atoms lying outside of the subsubdomain. This is consistent with the definition of volume used to define the density of molecules in the chemical potential, but it raises a problem for the parallel algorithm. First of all, the minimum thickness of the buffer layer, $\Delta_{s u b}$, must now be increased from $r_{s} / 2$ to $\left(r_{c o m}+r_{s} / 2\right)$ where $r_{c o m}$ is the farthest distance of any atom from its centroid. For a diatomic molecule this is half the bond-length. In addition, the minimum domain size must be increased from $2 r_{c}$ to $2 *\left(r_{c}+2 r_{c o m}-2 \Delta_{s u b}\right)$. Using the minimum domain size and the minimum buffer layer, the sub-subdomain size is then equal to $r_{c}-2^{*}\left(r_{c o m}-r_{s}\right)$. Assuming that $r_{s}$ is small, the sub-subdomain size will vanish for $r_{\text {com }}=r_{c} / 2$, or about $5 \AA$. Hence, for small molecules the scheme is viable, but it starts to break down as the length of the molecule increases. Ultimately, this situation could be resolved by enabling insertion and deletion of molecules which cross domain boundaries. This would also obviate the need for the $\Delta_{s u b}$ parameter.

\section{The Resulting Parallel DCV-GCMD Algorithm}

As before, the $G C$ phase is driven by a subroutine which is hooked into the LAMMPS MD step after the first velocity update. However, the driver subroutine only performs a $\mathrm{GC}$ sequence every $N_{\text {exchange }}$ timesteps. Otherwise it returns immediately to LAMMPS.

The first step in the GC sequence is a swap, where processors exchange ordered lists of position information for borrowed atoms; this is necessary because atoms have just 
completed a move. Next, indexed lists of exchangeable molecules with centroids lying in each sub-subdomain are compiled. The thermodynamic and statistical properties of the system are also sampled. Then $N_{c y c l e}$ GC cycles are performed.

Each GC cycle runs over the eight sub-subdomains lying within a given processor domain. If the sub-subdomain lies within one of the control volumes, a molecule insertion or deletion is attempted. The attempt is accepted with a probability based on the resultant energy change. The insertion/deletion attempt may be followed by an identity swap attempt, if requested. For sub-subdomains which lie outside the control volumes, a Widom insertion is performed to measure the chemical potential profile. If identity swaps have been requested for the control volumes, two additional Widom insertions are performed. After each attempted insertion, deletion or identity swap, any changes which have occurred are communicated to neighboring processors.

Upon completion of a GC cycle over the eight sub-subdomains, global tags are assigned to any newly created atoms, and these tags are communicated to neighboring processors. Upon completion of all $N_{\text {cycle }}$ GC cycles, the thermodynamic and statistical properties of the system are sampled again. Upon return to the LAMMPS code, a re-neighboring is performed only if a GC sequence has been performed.

\section{Chemical Potential Control for Large Molecules}

Because the accompanying change in energy of the insertion or deletion of large molecules is so great as to make the probability of accepting such a move prohibitively small, the range of applicability of all grand canonical simulation methods is limited, including the original DCV-GCMD method. To overcome this problem, special incremental insertion/ deletion schemes have been developed, such as the algorithm discussed above implemented in LADERA to enable the insertion/deletion of small rigid molecules.

Thus a second effort in the course of this work has been to overcome the limitations of such methods: not only are they computationally expensive, but they are applicable only to particular types of molecules. Our approach $[10,4]$ to solving this problem, is to avoid insertion/deletion of component $n$ altogether, so that the total the number of molecules of that component remains fixed. This amounts to performing a Legendre transform on the control volume simulations; we switch from constant- $\mu_{1} \ldots \mu_{n-1} \mu_{n} V T$ to constant- $\mu_{1} \ldots \mu_{n-1} N_{n} V T$ simulations. This creates a new problem, in that the diffusion of component $n$ will cause the intensive simulation parameter $N_{n}$ in each control volume to increase or decrease. The volume $V$, which is the only other extensive simulation parameter, remains fixed, and as a result, the thermodynamic state of each control volume changes with time. To prevent this, we perform a second Legendre transform, replacing the $V$ with its intensive conjugate $P$. This new constant $-\mu_{1} \ldots \mu_{n-1} N_{n} P T$ ensemble is the multicomponent analog of the isothermal-isobaric ensemble. The important feature is that $N_{n}$ is the only extensive fixed parameter. Hence as $N_{n}$ changes within a given control volume, the entire control volume can change with it. The thermodynamic state is determined only by the intensive fixed parameters, and so remains unchanged. 
Conventional affine expansion/contraction techniques for controlling system pressure [5] can not be used here. These methods are based on global changes to the system volume, whereas we require that independent volume changes occur in the left and right control volumes. In addition, these methods would not preserve the length of the region between the two control volumes, making it impossible to define a concentration gradient. Instead, we use a type of pressure control introduced by Lupkowski and van Swol [31] for equilibrium $\mathrm{MD}$ simulations of confined fluids. The Hamiltonian describing the system of interacting particles is augmented by the addition of two quasi-particles, labelled $A$ and $B$, which we will refer to as pistons. The sole purpose of the pistons is to enforce constantpressure boundary conditions independently at opposite ends of the simulation box. For this reason, the dynamics of the pistons can be greatly simplified. Each piston has fixed $y$ and $z$ coordinates, and a fixed orientation normal to the $x$-direction. Hence, it has only one degree of freedom: translation in the $x$-direction. The Newtonian equations of motion of the augmented system are given by:

$$
\begin{gathered}
m_{i} \ddot{\mathbf{r}}_{i}=\sum_{j \neq i}^{N} \mathbf{F}_{i j}\left(\mathbf{r}_{i j}\right)+\mathbf{F}_{i w}\left(\mathbf{r}_{i A}\right)+\mathbf{F}_{i w}\left(\mathbf{r}_{i B}\right) \\
m_{w} \ddot{\mathbf{r}}_{A}=P A_{y z} \mathbf{n}_{A}-\sum_{i=1}^{N} \mathbf{F}_{i w}\left(\mathbf{r}_{i A}\right) \\
m_{w} \ddot{\mathbf{r}}_{B}=P A_{y z} \mathbf{n}_{B}-\sum_{i=1}^{N} \mathbf{F}_{i w}\left(\mathbf{r}_{i B}\right)
\end{gathered}
$$

where $m_{i}$ is the mass of particle $i, \mathbf{r}_{i}$ is its position vector, and $\mathbf{F}_{i j}$ is the force on particle $i$ due to particle $j . m_{w}$ is the piston mass, $P$ is the desired system pressure, $A_{y z}$ is the crosssectional area of the system on the $y z$ plane, $\mathbf{r}_{A}$ and $\mathbf{r}_{B}$ are the positions of the pistons and $\mathbf{n}_{A}$ and $\mathbf{n}_{B}$ are unit vectors in the positive and negative $x$-directions, respectively. $\mathbf{F}_{i w}\left(\mathbf{r}_{i w}\right)$ is the force on particle $i$ at $\mathbf{r}_{i}$ due to a piston located at $\mathbf{r}_{w}$ where $\mathbf{r}_{i w}=\mathbf{r}_{i}-\mathbf{r}_{w}$ and is given by:

$$
\mathbf{F}_{i w}(\mathbf{r})=\frac{d}{d r} \phi_{i w}(r) \frac{\mathbf{r}}{r}
$$

where $\phi_{i w}(r)$ is the pair interaction energy between a particle $i$ and a piston at a distance $r$ away. It is clear from these equations, that the dynamics of the augmented system can be simulated by straight-forward extension of the standard methods of molecular dynamics simulation. In our parallel implementation, each processor calculates $\mathbf{F}_{i w}\left(\mathbf{r}_{i A}\right)$ and $\mathbf{F}_{i w}\left(\mathbf{r}_{i B}\right)$ for all the atoms which it owns. The total force on each piston is then calculated by summing over all processors. The piston positions are updated on processor 0 , and then communicated to the other processors. In addition, the kinetic energy of the pistons is included in the temperature-rescaling algorithm. 
Usually $\phi_{i w}(r)$ is chosen to be a short-ranged repulsive potential which prevents atoms from crossing through the pistons, while limiting the effect of the pistons to a layer of fluid which is only several atoms thick. The repulsive force of the fluid on the atoms is counteracted by the constant restraining force, $P A_{y z}$. As a result, once the system has equilibrated, the force exerted by the atoms on the piston will fluctuate about that of the constant restraining force, thus maintaining a constant average pressure in the $x$-direction in the fluid near the wall. As long as the system is not changing rapidly, this local pressure control will propagate throughout the system. Implicit in this approach is the assumption that the same pressure is imposed on both pistons, i.e. there is no pressure gradient in the system.

The use of the pistons in DCV-GCMD imposes certain constraints on how the simulation is conducted. Firstly, we can no longer have periodic boundaries in the $x$-direction, and so there is only one gradient zone, which passes through the center of the box. Secondly, the pistons are not allowed to penetrate the control volumes. This condition could probably be relaxed, but in our parallel implementation it simplifies the computation. Hence two buffer regions of fluid are set up between the starting positions of the pistons and the control volumes proper. As the simulation proceeds, component $n$ migrates across the simulation box, leaving one buffer region and accumulating in the other. Let us assume that this migration is from left to right. As a result, the pistons will also tend to undergo net motion from left to right, causing the left buffer to shrink and the right buffer to grow. Eventually, the left piston will reach the left control volume, at which point the simulation must be terminated. Hence the maximum duration of the simulation is limited by the size of the lefthand buffer. An additional constraint is imposed by the spatial domain decomposition. The right buffer may eventually grow to a point where it extends beyond the range of the rightmost processor domain. In this case the simulation must also be terminated. Despite the transient nature of the simulation, the two control volumes and the central gradient zone form a subsystem which is maintained at steady state, thus allowing transport properties to be measured.

\section{Applications of the DCV-GCMD and Extended DCV-GCMD Methods}

\section{Diffusion in Polymers}

The extension of the LADERA code to the diffusion of single-site penetrants through bonded macromolecules $[11,21]$ was initially prototyped with a variation of the simple WCA color diffusion system studied in [1], systems B1 and B2, discussed above. The density profiles for all species in system $B 1$ as a function of $x$-position are shown in Figure 6. These are the profiles from a run on 256 processors in a $16 \times 4 \times 4$ ( $x y z)$ array, averaged over 150,000 steps after 325,000 steps of equilibration, starting from a box with only equilibrated polymer. This is for the case of $r_{s}=0.5 \sigma$ and $\Delta_{\text {sub }}=0.25$. The shaded regions represent the control volumes, with the boundaries moved in by $\Delta_{\text {sub. }}$. Note that the profile for species 3 is shown as a bead density, rather than a molecule density. Although there are some fluctuations in the profiles of species 1 and 2 at higher concentrations in the control 
Gradient-Driven Diffusion of Multi-Atom Molecules Through Macromolecules and Membranes: LDRD 96-0021 Close-out Report

volumes, the densities are essentially constant across them, and smooth linear profiles are observed in between. Since the gradients of these two species are symmetric, there is actually no overall pressure gradient in the system, and therefore the polymer beads are distributed uniformly throughout the entire simulation box. The magnitude of the observed fluctuations can be arbitrarily reduced with longer runs. We fit the diffusion behavior of species 1 and 2 to Fick's Law in one dimension,

$$
J_{i}=-D_{i} \frac{\partial \rho_{i}}{\partial x},
$$

where $J_{i}$ and $D_{i}$ are the flux and diffusivity of species $i$ in the mixture, respectively. The $J_{i}$, as calculated by the control volume method [1], are given in Table 2 .

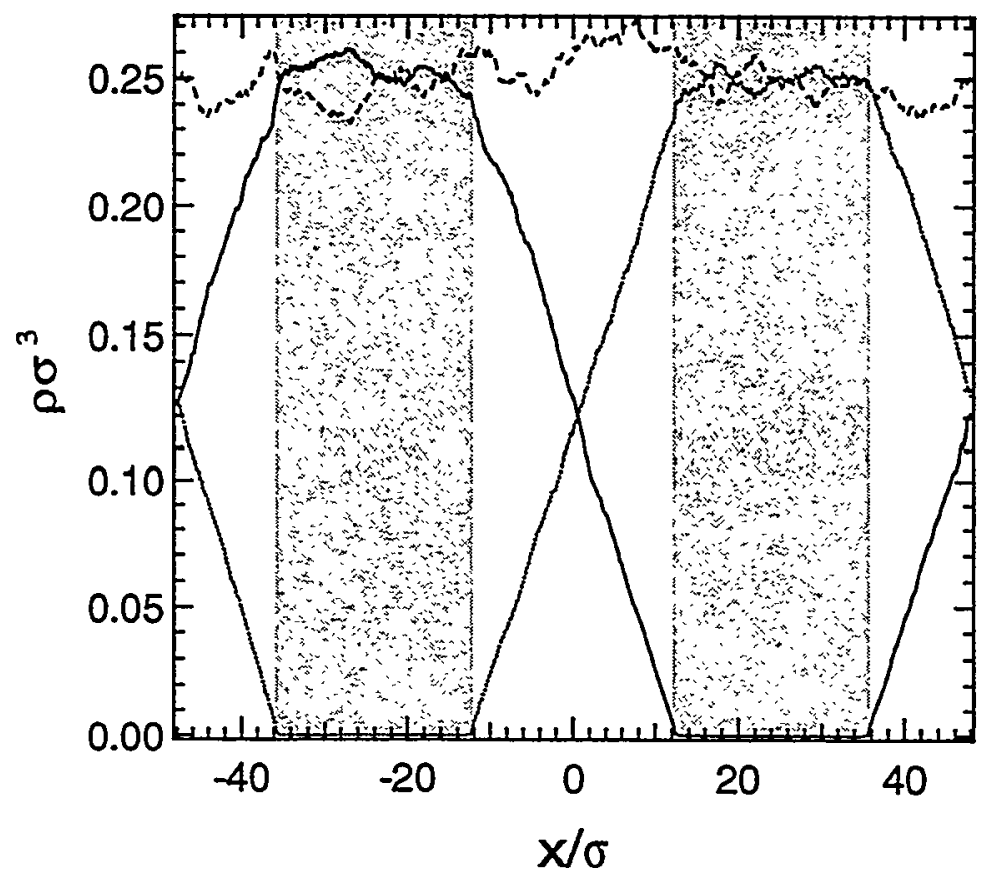

Figure 6. Density profiles for all species in system B1 as a function of $x$-position. The solid line is for species 1 , the dotted line is for species 2 , and the dashed line is for species 3 (shown as a bead density). The shaded regions represent the control volumes, with the boundaries moved in by $\Delta_{\text {sub }}$. The value of $r_{s}$ is $0.5 \sigma$ and the value of $\Delta_{\text {sub }}$ is $0.25 \sigma$.

Table 2. Flux Data for System B1

\begin{tabular}{|c|c|c|c|}
\hline Species & $J_{i} \sigma^{3}(m / \varepsilon)^{1 / 2}$ & $\mathrm{~d}\left(\rho_{i} \sigma^{3}\right) / \mathrm{d}(x / \sigma)$ & $D_{i}(m / \varepsilon)^{1 / 2} / \sigma$ \\
\hline 1 & 0.0028 & -0.00984 & 0.28 \\
\hline 2 & -0.0026 & 0.00934 & 0.28 \\
\hline
\end{tabular}


The quantities $\partial \rho_{\mathrm{i}} / \partial x$ are also given in the table; these were obtained from a fit to the central portion of the simulation box, as shown in Figure 7. The diffusion coefficients resulting from application of eqn 12 are given as well; the $D_{i}$ of species 1 and 2 are identical, as they should be. It is interesting to compare this value of the diffusivity with that in a pure monomer fluid at the same total segment density $\left(r_{1}+r_{2}+r_{\text {bead }}\right)$. This diffusivity is available from the previous DCV-GCMD study of Heffelfinger and van Swol [1], where it was found to be 0.28 . This value is identical to that in the present study, suggesting that the degree of bonding in the system has very little effect on the diffusivity. This finding is in agreement with some recent work by Sanchez on hard-sphere diffusants in hard-sphere chain polymers [32]. This behavior will probably not be seen with more complex polymer models, where internal motions of the polymer (e.g. dihedral rotations) may be strongly coupled to penetrant diffusion.

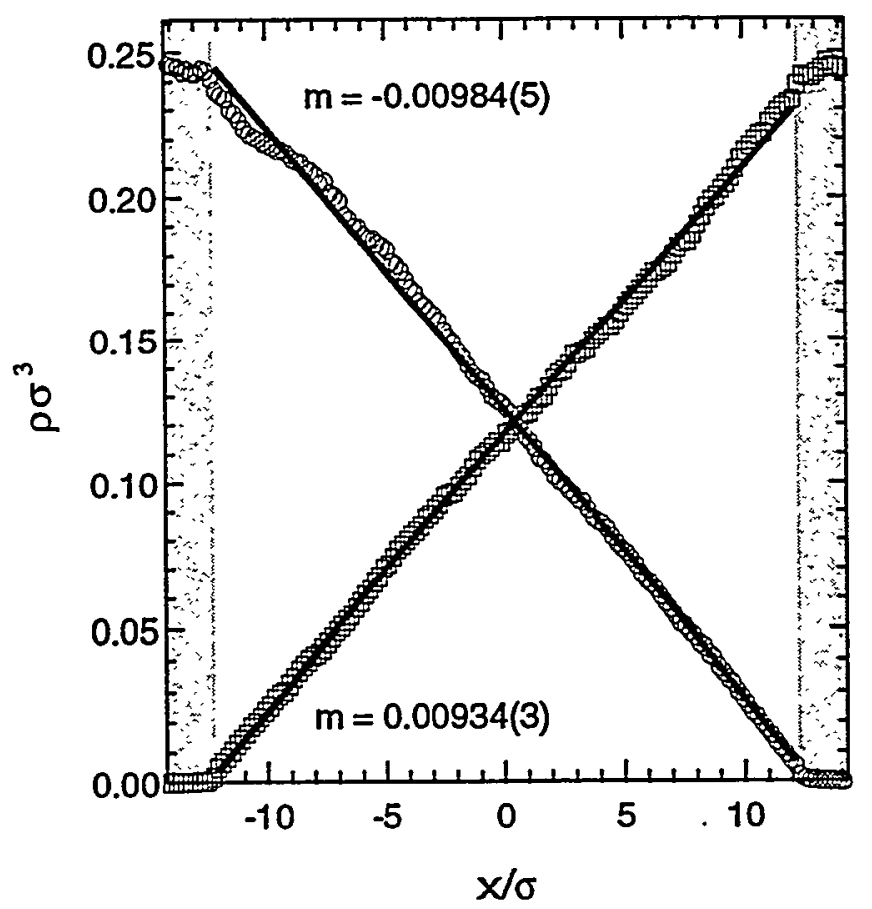

Figure 7. The central region of Figure 6. The symbols represent the actual simulation data; the circles are for species 1 and the squares are for species 2 . The solid lines are the linear least-squares fit to each profile in this region. The labels are the slopes of these lines. The error in the last decimal place of the slope is given in parentheses.

Figure 8 is a plot of the density profiles for System B2. This is the profile from a run on 64 processors in a $4 \times 4 \times 4(x y z)$ array, averaged over 680,000 steps after 100,000 steps of equilibration, starting from a box with only equilibrated polymer. This plot is for the case of $r_{s}=2.0 \sigma$ and $\Delta_{\mathrm{sub}}=1.0 \sigma$; these are both rather large values which are probably far from optimal. As in Figure 6, the shaded regions represent the control volumes, with the boundaries moved in by an increment $\Delta_{\text {sub }}$. The profiles seem to exhibit greater noise than those in Figure 6, but this is primarily due to the much smaller scale of the abscissa. Two 
other striking features in Figure 8 are the rounded edges in the density profiles of species 1 and 2 near the control volume boundaries and the failure of the densities to reach exactly zero, even at the centers of the control volumes. These features are due to the large value of $\Delta_{\text {sub }}$ used here, which leads to a large amount of dead space in the control volumes. In fact, with the subdomain dimensions used here $(3 \sigma \times 3 \sigma \times 3 \sigma)$, the fraction of dead space in the domains was $96 \%$ (compared to $42 \%$ for Figure 6 ). With this much dead space, it is somewhat amazing that the profiles are as distinct as they are.

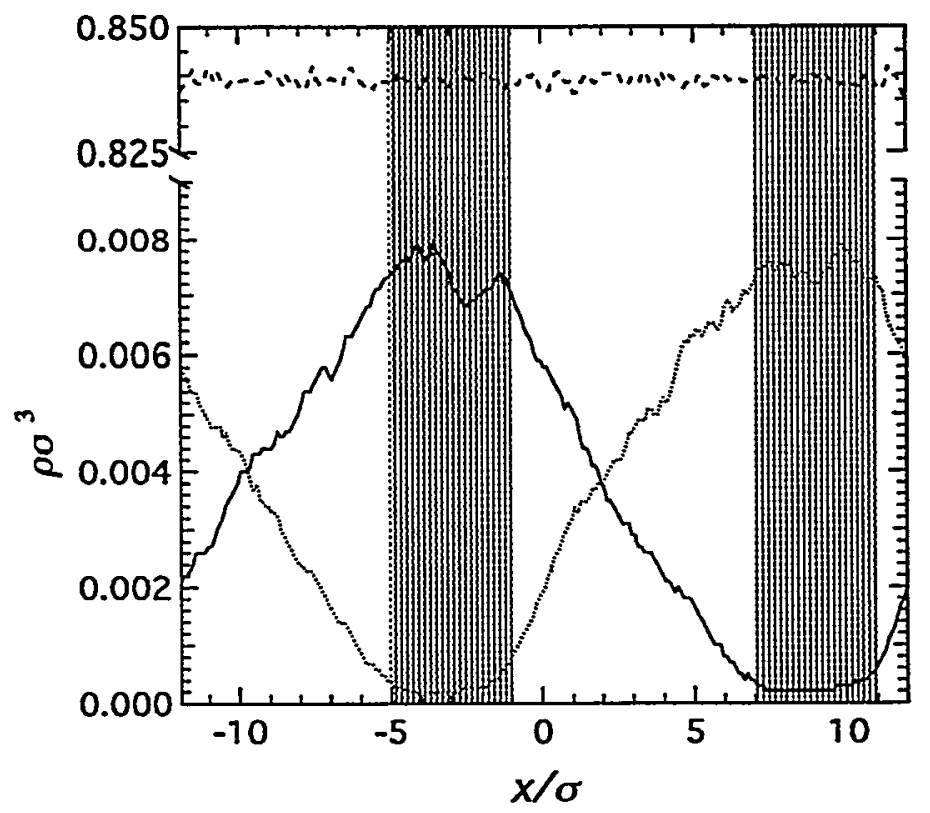

Figure 8. Density profiles for all species in system B2 as a function of $x$-position. The solid line is for species 1 , the dotted line is for species 2 , and the dashed line is for species 3 (shown as a bead density). The shaded regions represent the control volumes, with the boundaries moved in by $\Delta_{\text {sub }}$. The value of $r_{s}$ is $2.0 \sigma$ and the value of $\Delta_{\text {sub }}$ is $1.0 \sigma$.

Although the profiles were not ideal, we performed a diffusion coefficient calculation by same method described above; see Table 3 and Figure 9 for details. The resulting values of $D_{i}$ compare favorably to the value of 0.045 obtained from long-time $\mathrm{MD}$ simulations by Plimpton and McCoy [33]. The above results for systems B1 and B2 demonstrate that a good estimate for permeation rate may be obtained from the new LADERA even for relatively short simulations and very dense polymers, suggesting that the new LADERA would be useful as a screening tool for polymers proposed for gas separation.

\section{Constant Pressure DCV-GCMD}

As discussed above, the success of the DCV-GCMD method in modeling systems experiencing steady-state chemical potential gradients is predicated on being able to adjust $N_{i}$, the number of molecules of species $i$, the species for which the gradient is desired. This 
limitation of the method has been partially relaxed by enabling control of the system pressure in the LADERA code via two moveable pistons. As an initial test of this new constant pressure DCV-GCMD method, a comparison with the original work of Lupkowski and van Swol [31], who first developed the pistons method of pressure control for confined equilibrium fluids, was carried out $[4,34]$ in several stages as follows.

Table 3. Flux Data for System B2

\begin{tabular}{|c|c|c|c|}
\hline Species & $J_{i} \sigma^{3}(m / \varepsilon)^{1 / 2}$ & $\mathrm{~d}\left(\rho_{i} \sigma^{3}\right) / \mathrm{d}(x / \sigma)$ & $D_{i}(m / \varepsilon)^{1 / 2} / \sigma$ \\
\hline 1 & 0.000045 & -0.00083 & 0.054 \\
\hline 2 & -0.000046 & 0.00078 & 0.059 \\
\hline
\end{tabular}

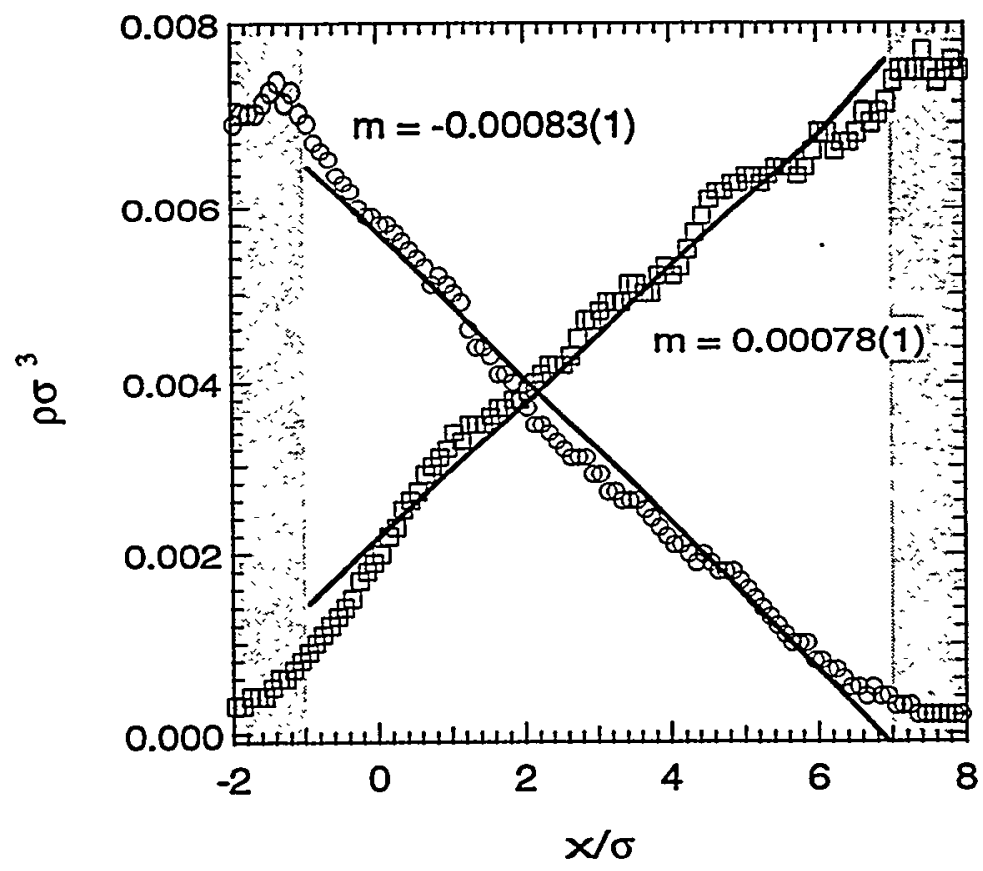

Figure 9. The central region of Figure 8. The symbols represent the actual simulation data; the circles are for species 1 and the squares are for species 2 . The solid lines are the linear least-squares fit to each profile in this region. The labels are the slopes of these lines. The error in the last decimal place of the slope is given in parentheses.

Validating the Piston LADERA Code as a NPTMD Code

The LADERA code modified with pistons for pressure control was first investigated by applying it as an NPTMD code with a pure WCA fluid. The interaction between the fluid 
atoms and the piston walls (fw interaction) was defined to be the WCA analog of the 9-3 potential:

$$
\phi_{f w}= \begin{cases}\varepsilon_{f w}\left[\frac{2}{15}\left(\frac{\sigma_{f w}}{x}\right)^{9}-\left(\frac{\sigma_{f w}}{x}\right)^{3}\right]+\varepsilon_{f w} \frac{\sqrt{10}}{3}, & x<(2 / 5)^{1 / 6} \sigma_{f w}, \\ 0, & x \geq(2 / 5)^{1 / 6} \sigma_{f w}\end{cases}
$$

where $\sigma_{\mathrm{fw}}$ describes the range and $\varepsilon_{\mathrm{fw}}$ the stiffness of the repulsive potential. Because the fluid-wall parameters $(f w)$ were set to those of the fluid-fluid interaction $(f)$, all values of $\sigma$ and $\varepsilon\left(\sigma_{\mathrm{ff},} \sigma_{\mathrm{fw}}, \varepsilon_{\mathrm{ff}}\right.$, and $\left.\varepsilon_{\mathrm{fw}}\right)$ are henceforth referred to simply as $\sigma$ and $\varepsilon$. The pressure was set to $\rho \sigma^{3} / \varepsilon=6.255$.

The temperature was maintained at $\mathrm{kT} / \varepsilon=1.5$ using a simple velocity rescaling algorithm. In the $x$-direction, the system was bounded by the pistons, but was free to expand and contract. In the $y$ and $z$ directions the dimensions were $12 \sigma$ and the boundary conditions were periodic. The number of atoms was fixed at 4961 , to give the same density per unit piston area as existed in Lupkowski's simulation, although they only used 160 atoms and had a correspondingly small box width of $4.31 \sigma$. The $M D$ simulation was run on a $4 \times 8 \times 8$ array of processors, using a timestep of $0.005\left(\varepsilon / m \sigma^{2}\right)^{1 / 2}$. The pistons masses were set to $10 m_{f}$. The starting configuration for this $N P T$ molecular dynamics simulation was created by equilibrating a $\mu \mathrm{VT}$ simulation with the wall positions fixed at $\pm 6.385 \sigma$. A configuration of this system was selected which contained exactly 4961 particles. The $N P T$ molecular dynamics simulation was then started from this configuration and run for 60,000 timesteps. The piston positions were sampled every 100 timesteps. The piston trajectories are shown in Figure 10. The system drifted slowly to the right, due to the fact that the total momentum of the system was not explicitly set to zero when the initial particle velocities were chosen from the Maxwell distribution. However, the separation between the walls fluctuated about a steady average value of $12.75 \sigma \pm 0.001$. This was in close agreement with Lupkowski and van Swol's value of $12.78 \sigma$.

\section{Color Diffusion}

In order to test the performance of the pistons in the context of gradient-driven diffusion, we applied the method to the binary system previously modeled by Heffelfinger and van Swol [1] and discussed above. As before, components 1 and 2 were represented by the WCA potential described above with identical values of $\sigma$ and $\varepsilon$, so that they differed only in their "color" or type. In addition, the fluid-wall interaction (component "w") received the same treatment, that is,

$$
\begin{gathered}
\sigma_{11}=\sigma_{22}=\sigma_{12}=\sigma_{1 w}=\sigma_{2 w} \equiv \sigma, \\
\varepsilon_{11}=\varepsilon_{22}=\varepsilon_{12}=\varepsilon_{1 w}=\varepsilon_{2 w} \equiv \varepsilon, \text { and }
\end{gathered}
$$




$$
m_{1}=m_{2} \equiv m_{f}
$$

The advantage of doing this type of "color diffusion" experiment is that the transport diffusivities obtained from the DCV-GCMD simulation can be compared directly with the self-diffusivity of a pure WCA fluid at the same overall density.

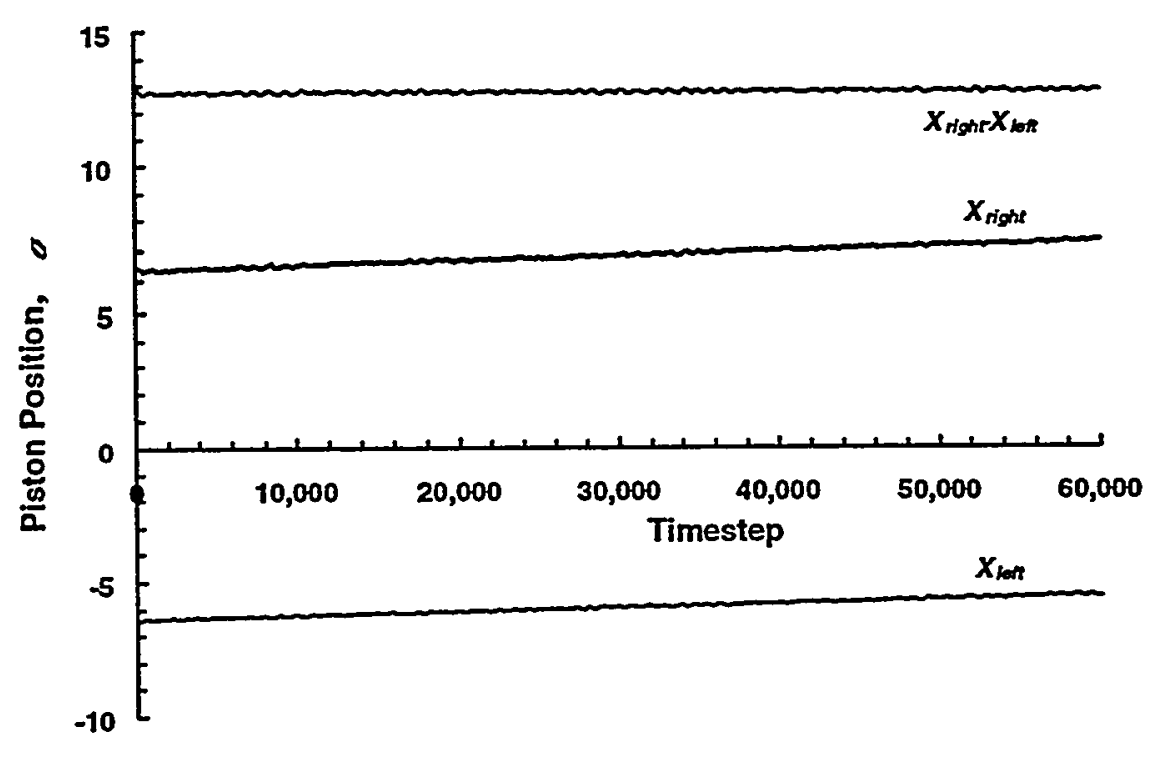

Figure 10. Plot of piston positions versus time for an NPT molecular dynamics simulation of a pure WCA fluid. The temperature was $1.5 \varepsilon / \mathrm{k}$, the pressure was $6.255 \varepsilon / \sigma^{3}$, the number of atoms 4961 . The lines are (from bottom) the left piston position, the right piston position, and the piston separation.

This system was first simulated using a standard DCV-GCMD simulation (constant $\mu_{1}, \mu_{2}$, $T, V$, which we will refer to as the constant-volume simulation. The temperature was maintained at $\mathrm{kT} / \varepsilon=1.0$ using velocity rescaling. The chemical potentials of the two components, $\mu_{1}^{\prime} / \varepsilon$ and $\mu_{2}^{\prime} / \varepsilon$, were maintained at 1.54 and 2.34 in control volume $A$ and 2.34 and 1.54 in control volume $B$. Thus a concentration gradient was established, while the overall density was maintained at a constant value throughout the system.

The constant-volume simulation was carried out on an $8 \times 2 \times 2$ processor grid. The system extended from $-24 \sigma$ to $24 \sigma$ in the $x$-direction and from $-6 \sigma$ to $6 \sigma$ in the $y$ and $z$ directions. The control volumes were located on the ranges $-18 \sigma<x<-6 \sigma$ and $6 \sigma<x<18 \sigma$. The $\mathrm{MD}$ timestep was $0.01\left(\varepsilon / m \sigma^{2}\right)^{1 / 2}$. The simulation was started from an empty box and was equilibrated for 20,000 timesteps. After equilibration, the simulation was run for 200,000 timesteps, during which the concentration and flux profiles were sampled. The 
Gradient-Driven Diffusion of Multi-Atom Molecules Through Macromolecules and Membranes: LDRD 96-0021 Close-out Report

calculation was carried out on 32 processors of an Intel Paragon MIMD parallel machine. Each timestep required about 0.1 seconds.

The control volume concentrations including density profiles and overall density agreed closely with those of Heffelfinger and van Swol [1] although different simulation box dimensions were used so that the concentration gradients can not be compared directly. The average concentration gradients and fluxes are given in Table 4 . The corresponding transport diffusivities have been calculated using the relation $D_{i}^{x}=-J_{i}^{x} /\left(d \rho_{i} / d x\right)$, which is valid for systems which are at rest relative to the stationary reference frame. All quantities were calculating using the methodology of Heffelfinger and van Swol [1]. The individual estimates of transport diffusivity lay in the range 0.27 to $0.36(\mathrm{~m} / \varepsilon)^{-1 / 2} \sigma$, in close agreement with Heffelfinger and van Swol's values [1], which lay in the range 0.27 to $0.34(m / \varepsilon)^{-1 / 2} \sigma$.

Table 4. Constant-volume DCV-GCMD simulation results

\begin{tabular}{|c|c|c|c|c|c|}
\hline \multirow{2}{*}{$\begin{array}{c}\text { Compo- } \\
\text { nent }\end{array}$} & \multirow{2}{*}{$\sigma^{4} \mathrm{~d} \rho_{\mathrm{i}} / \mathrm{dx}$} & \multicolumn{2}{|c|}{ Control Volume } & \multicolumn{2}{c|}{ Flux Plane } \\
\cline { 3 - 6 } & & $\mathrm{J}_{\mathrm{i}}^{\mathrm{x}} \sigma^{3}(\mathrm{~m} / \varepsilon)^{1 / 2}$ & $\mathrm{D}_{\mathrm{i}}^{\mathrm{x}}(\mathrm{m} / \varepsilon)^{1 / 2} / \sigma$ & $\mathrm{J}_{\mathrm{i}}^{\mathrm{x}} \sigma^{3}(\mathrm{~m} / \varepsilon)^{1 / 2}$ & $\mathrm{D}_{\mathrm{i}}^{\mathrm{x}}(\mathrm{m} / \varepsilon)^{1 / 2} / \sigma$ \\
\hline 1 & 0.0124 & 0.00447 & 0.360 & 0.00451 & 0.364 \\
\hline 2 & 0.0122 & 0.00331 & 0.271 & 0.00334 & 0.274 \\
\hline Average & 0.0123 & 0.00389 & 0.314 & 0.00393 & 0.319 \\
\hline
\end{tabular}

The final flux profiles, calculated via the $x$-direction velocity averaging method discussed above, are shown in Figure 11. The fluxes in the positive $x$-direction are taken to be positive. Hence, the flux of component 2 is positive in the center of the simulation box and negative at the edges. The noise in the flux profiles is found primarily within the control volumes because it is here that insertions and deletions are carried out. In the "gradient zones" (i.e. the two regions connecting the control volumes), the fluxes are very smooth but non-zero. One would expect zero fluxes in the control volumes due to the lack of a concentration gradient. However, for this to occur, a much larger number of insertions and deletions would be needed. In practice, both the concentration gradients and the fluxes change rapidly near the boundaries, and are relatively flat in the center of the control volumes. Thus the flux profiles vary continuously from the value of the flux in one gradient zone, through zero, to the value of the flux in the other gradient zone. In the limit of an infinite number of GCMC cycles per MD timestep, the relative flux within each control volume would be zero and there would be a discontinuity at each control volume boundary. (The same would be true of the concentration gradients.)

We also see from this figure that magnitude of the flux for both components varies outside the gradient zones but is constant within each gradient zone. Constant species fluxes in the gradient zones define the existence of steady-state. However, the magnitude of the gradient 
zone flux fluctuates around an average value as the simulation proceeds. It is this fluctuation which is responsible for the fact that the flux profiles for a given species in Figure 11 do not have equal and opposite values in the two gradient zones. However, we can see that the values of the relative flux, $J_{1}^{x}-J_{2}^{x}$, in the two gradient zones are almost exactly equal and opposite. This illustrates the fact that it is the relative flux which is directly determined by the concentration gradient, rather than the individual species fluxes.

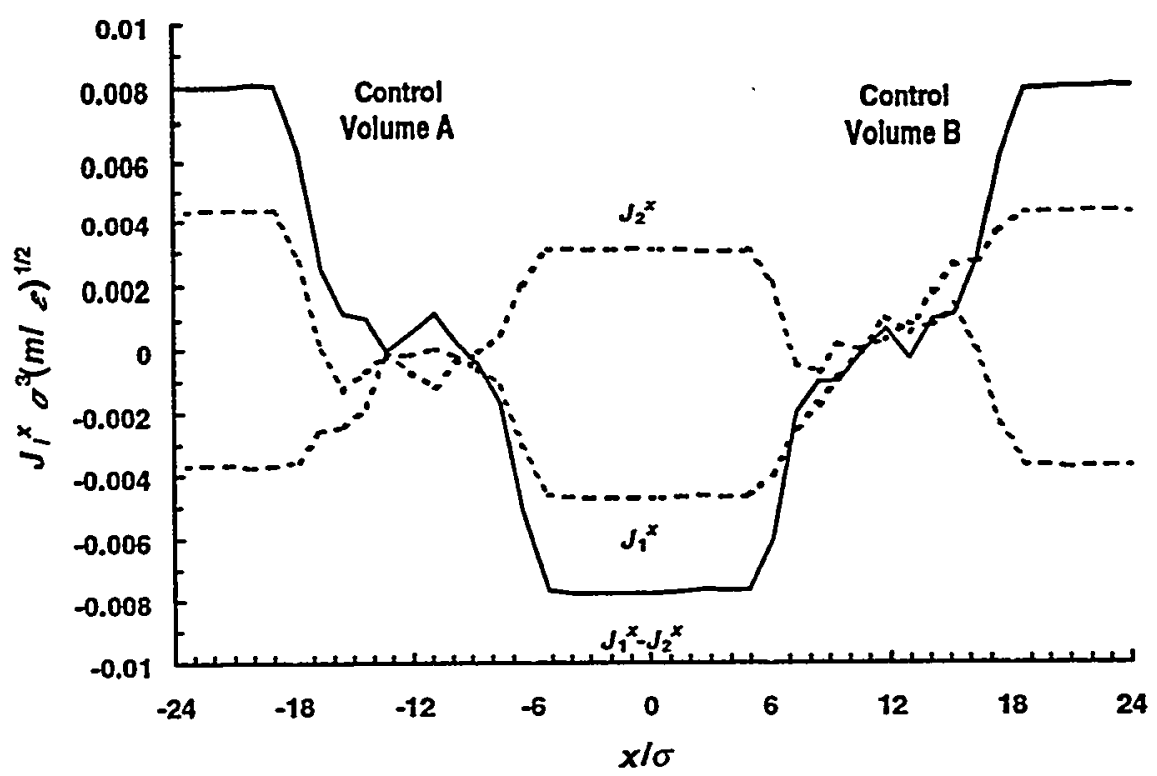

Figure 11. Average flux profiles for the constant-volume DCV-GCMD simulation. The dashed lines are the absolute fluxes, measured relative to the "stationary coordinate reference frame", i.e. the simulation box coordinates. The solid line is the flux of component 1 relative to component 2 and is an approximation to the true diffusion flux.

Having established a benchmark using constant-volume DCV-GCMD, we then simulated the same system at constant pressure using piston pressure control instead of inserting and deleting component 2 (constant $\mu_{1}, N_{2}, T, P$ ). We will call this the constant-pressure simulation. The simulation was carried out on an $18 \times 4 \times 4$ processor grid. The simulation box extended from $-54 \sigma$ to $54 \sigma$ in the $x$-direction and from $-12 \sigma$ to $12 \sigma$ in the $y$ and $z$ directions. The simulation was started from an empty box with fixed walls located at $\pm 36 \sigma$. To quickly equilibrate the system, the control volumes were temporarily extended out to the ends of the simulation box, and both species were inserted and deleted, using the same chemical potentials as before. Steady-state was achieved after 5,000 timesteps. At this point, the system contained 9578 species 1 atoms and 9024 species 2 atoms. From this configuration, the constant-pressure simulation was executed for 300,000 timesteps. The pressure was set at $1.4 \sigma^{3} / \varepsilon$ (equal to the average pressure from the constant-volume 
Gradient-Driven Diffusion of Multi-Atom Molecules Through Macromolecules and Membranes: LDRD 96-0021 Close-out Report

simulation). The piston masses were set to $3 m_{f}$. The control volumes coordinates were reset to those used in the constant-volume simulation. While the number of atoms of component 1 was allowed to vary through insertions and deletions in the control volumes to achieve a chemical potential of component 1 of 1.54 in control volume $A$ and 2.34 in control volume $B$, the number of atoms of component 2 remained fixed at 9024 . The calculation was carried out on 288 processors of an Intel Paragon MIMD parallel machine with each timestep requiring about 0.1 seconds, the same as for the constant-volume simulation. This is to be expected, as the presence of the pistons does not add much to the computational effort, and the total number of insertions and deletions per timestep is the same for both simulations.

The evolution of the concentration profile during consecutive 20,000 timestep intervals for component 2 is contained in Figure 12. For clarity, all curves except the bottom one are shifted upwards in increments of $\rho \sigma^{3}=0.1$. The figure clearly demonstrates how the left buffer shrinks, the right expands, and the central portion of the simulation box does not change. The right buffer actually grows faster than the left buffer shrinks because the density of component 2 is lower on the right. The characteristic layering of the atoms at the piston walls are somewhat apparent, although the movement of the pistons tends to blur this detail. The simulation actually terminated at timestep 291170 , when the right piston position exceeded $54 \sigma$.

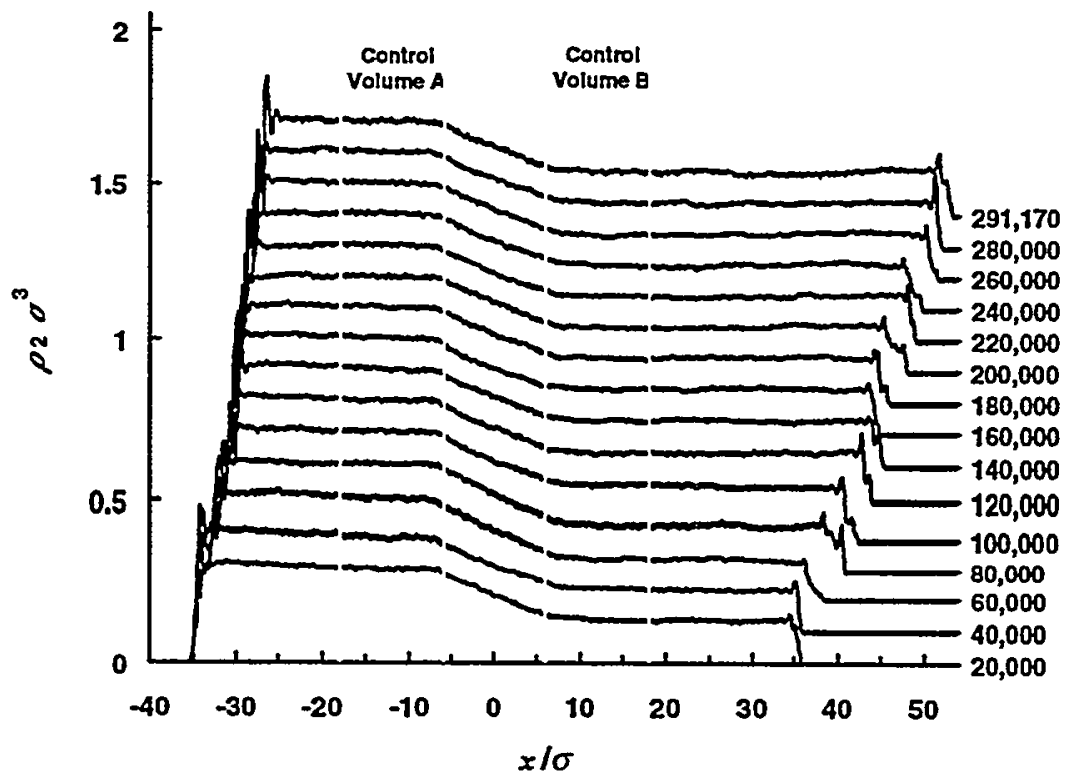

Figure 12. Time evolution of the concentration profile for component 2 in the constantpressure DCV-GCMD simulation. Each line represents the average concentration profile accumulated over 20,000 timesteps; plot labels indicate the last timestep sampled. For clarity all the lines except the lowest have been offset vertically by successive increments of $\rho \sigma^{3}=0.1$. 
The density and flux profiles sampled between timesteps 100,000 and 200,000 of the simulation are shown in Figure 13 and Figure 14. The central portions of the concentration profiles from the constant-volume simulation have been included for comparison. The agreement was close, but the constant-pressure simulations suffered a little more from "bleeding" at the control volume boundary, and consequently exhibited a slightly smaller concentration gradient. Also, the constant-pressure concentration profiles are slightly nonlinear. This is a consequence of the fact that the net flux $\left(J_{1}^{x}+J_{2}^{x}\right)$ in the gradient zone is no longer zero, as will be explained below.

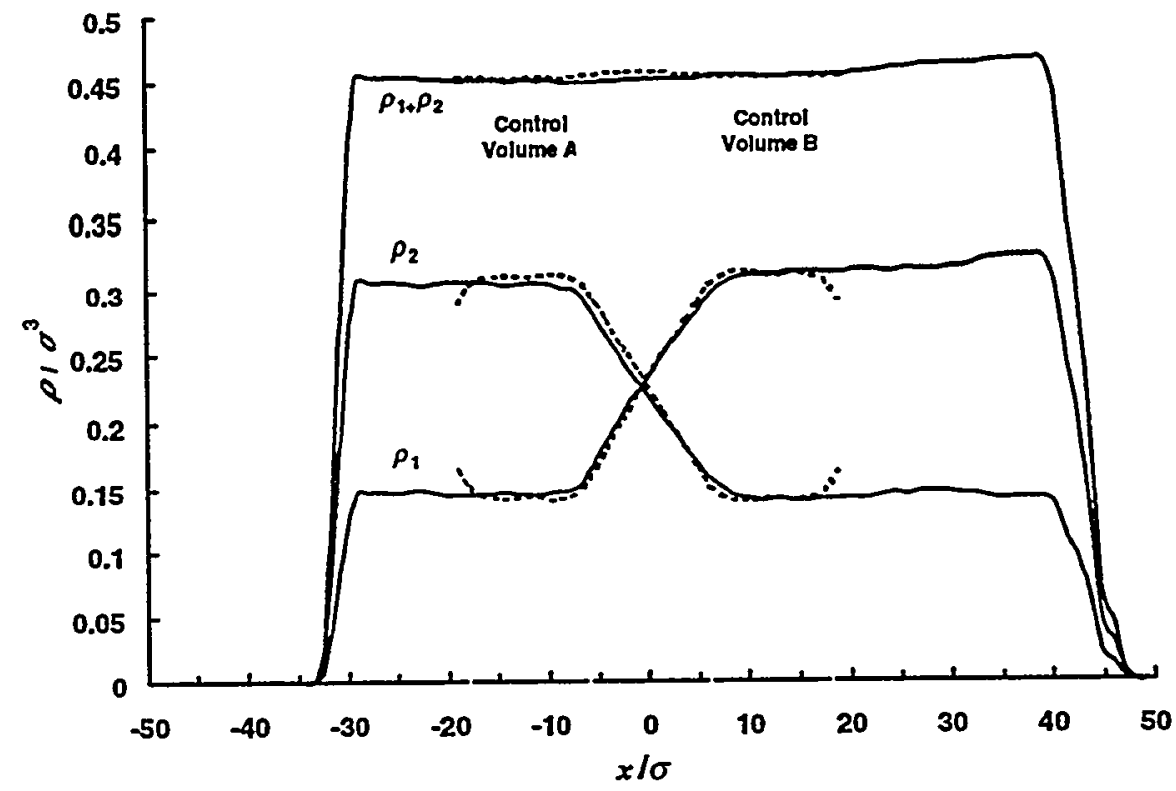

Figure 13. Average concentration profiles sampled between timesteps 100,000 and 200,000 of the constant-pressure DCV-GCMD simulation (solid lines). The dashed lines are the profiles from the central region of the constant-volume DCV-GCMD simulation.

The component flux profiles differ dramatically from those obtained previously. In the buffer zones, both components are flowing in the positive $x$-direction. This behavior is due to the bulk motion of the pistons from left to right. In the gradient zone, component 1 is diffusing strongly in the negative $x$-direction, while the flux of component 2 is roughly constant throughout the box. This raises to important questions:

- How can the diffusion coefficient be determined from the flux and concentration profiles?

What factors control the fluxes in the various parts of the simulation box?

We can best answer the former question by first of all addressing the latter. First of all, we need to replace the absolute fluxes $J_{i}^{x}$ by the more general diffusive fluxes $J_{i D}{ }^{x}$, which are given by [35], 
Gradient-Driven Diffusion of Multi-Atom Molecules Through Macromolecules and Membranes: LDRD 96-0021 Close-out Report

$$
J_{i D}^{x}=J_{i}^{x}-x_{i} \sum_{i=1}^{n} J_{i}^{x},
$$

where $x_{i}$ is the mole fraction of component $i$. In this case, Fick's Law is written as

$$
\frac{d \rho_{i}}{d x}=-\frac{J_{i D}^{x}}{D_{i}^{x}}=\frac{J_{t o t}^{x}}{D_{i}^{x} \rho_{t o t}} \rho_{i}-\frac{J_{i}^{x}}{D_{i}^{x}}
$$

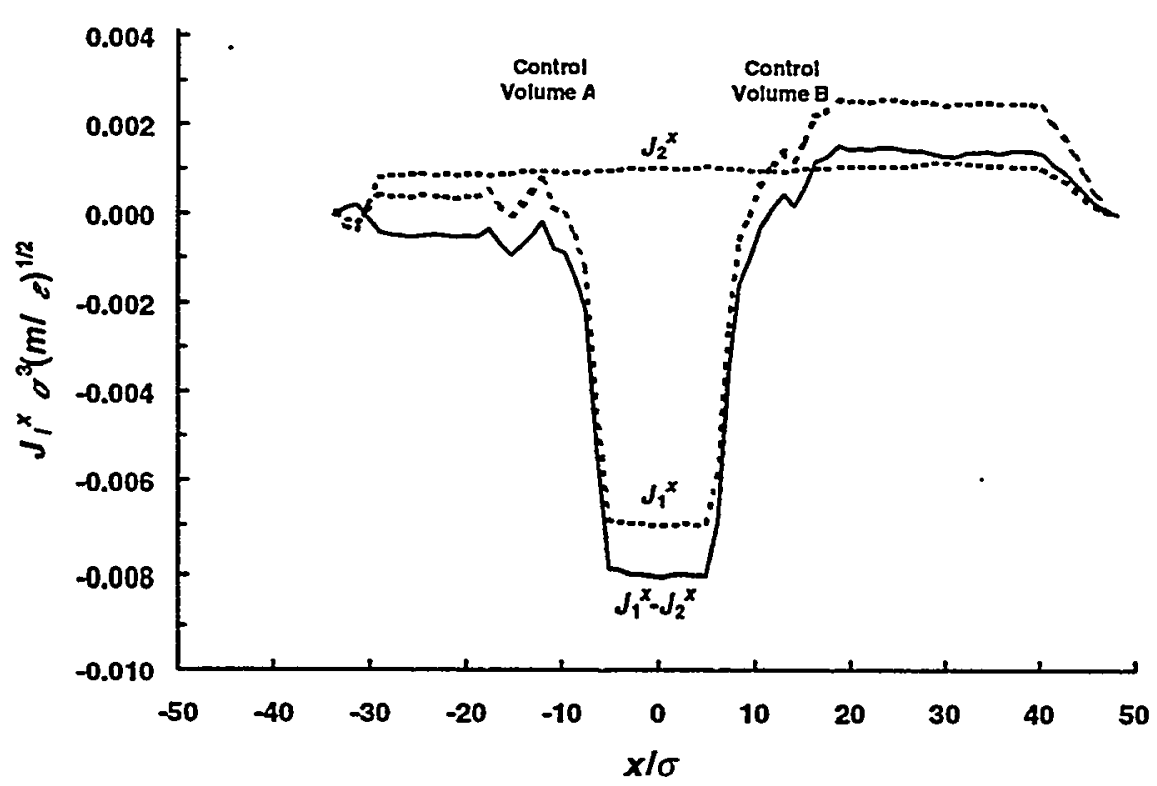

Figure 14. Average flux profiles sampled between timesteps 100,000 and 200,000 of the constant-pressure DCV-GCMD simulation. The dashed lines are the absolute fluxes, measured relative to the "stationary coordinate reference frame", i.e. the simulation box coordinates. The solid line is the flux of component 1 relative to component 2 and is an approximation to the true diffusion flux.

In general, eqn 18 must be integrated numerically. However, in the case of color diffusion, where the diffusion coefficients and total density are constant, the equation can be solved analytically. The solution is an exponential profile [4]:

$$
\left(\frac{\rho_{i}(x)-\rho_{i}^{\infty}}{\rho_{i}^{A}-\rho_{i}^{\infty}}\right)=\left(\frac{\rho_{i}^{B}-\rho_{i}^{\infty}}{\rho_{i}^{A}-\rho_{i}^{\infty}}\right)^{\frac{x-x_{A}}{x_{B}-x_{A}}}
$$

where 


$$
\rho_{i}^{\infty}=\frac{J_{i}^{x} \rho_{t o t}}{J_{t o t}^{x}}
$$

For zero total flux, the parameters $\rho_{i}^{\infty}$ are infinite and eqn 19 becomes linear. However, in general, the $\rho_{i}^{\infty}$ are finite and are determined by some external constraint on the system, which in the case of our simulation is the requirement that momentum be conserved. This is one example of the general concept of the bootstrap condition in diffusion experiments [35]. The full momentum balance is a little complicated in the piston simulation, but we can simplify it by making some reasonable assumptions:

1. The simulation is in a quasi-steady state, in that the "macroscopic" descriptors of the system change much more slowly than local fluctuations in density, momentum, etc.

2. The simulation box is divided into three distinct regions: the left buffer and control volume $A$, with total length $L_{A}$, the gradient zone, of length $L_{G}$, and the right buffer and control volume $B$, with total length $L_{B}$. These dimensions are treated as constant.

3. The fluxes do not change with time (steady-state).

4. The flux of component two $J_{2}^{x}$ is constant throughout the simulation box (steadystate).

5. The concentrations of the two components are constants in the left control volume and buffer zone $\left(\rho_{i}^{A}\right)$ and in the right control volume and buffer zone $\left(\rho_{i}^{B}\right)$.

6. As a consequence of assumptions 4 and 5 , the flux of component one is constant in the left control volume and buffer zone $\left(J_{1}^{A x}\right)$ and in the right control volume and buffer zone $\left(J_{1}^{B x}\right)$.

7. Insertion and deletion of component 1 has no net effect on the momentum of the system. In fact, the effect should be to add momentum in the opposite direction to the diffusion of component 1 , since particles are added with zero momentum on average but particles which are removed will tend to be moving in the direction of $J_{1}^{x}$.

With these assumptions, the conservation of momentum constraint results in the following expression for $J_{2}^{\chi}$ in a piston simulation involving particles of different mass:

$$
J_{2}^{x}=\frac{\left(m_{1} J_{1}^{G x}+m_{2} J_{2}^{x}\right) L_{G}}{\frac{P_{t o t}^{x}}{A J_{2}^{x}}-\frac{L_{A}}{\rho_{2}^{A}}\left(m_{1} \rho_{1}^{A}+m_{2} \rho_{2}^{A}\right)-\frac{L_{B}}{\rho_{2}^{B}}\left(m_{1} \rho_{1}^{B}+m_{2} \rho_{2}^{B}\right)} .
$$


Gradient-Driven Diffusion of Multi-Atom Molecules Through Macromolecules and Membranes: LDRD 96-0021 Close-out Report

In this equation, $P_{\text {tot }}^{x}$ is the total linear momentum in the $x$-direction, $A$ is the cross-sectional area of the box, and $m_{1}$ and $m_{2}$ are the masses of the two components. In the case of the color diffusion simulation, where the particles are of equal mass, and the total momentum is initially zero, this equation simplifies to an explicit expression for $\rho_{2}^{\infty}$ :

$$
\rho_{2}^{\infty}=\frac{J_{2}^{x} \rho_{t o t}}{J_{t o t}^{G x}}=-\frac{L_{G}}{L_{A} / \rho_{2}^{A}-L_{B} / \rho_{2}^{B}}
$$

The physical meaning of this equation is that the motion of the system from left to right due to the diffusion of component 2 must be balanced by an opposing motion from right to left within the gradient zone, due to the diffusion of component 1 . The smaller the gradient zone, relative to regions $A$ and $B$, the larger must be the flux. Hence, in the limit of very large buffer zones, the mass of the gradient zone becomes negligible, and the buffer zones must have zero flux. In this limit, the pistons do not move. In fact, the only non-zero flux is that of component 1 in the gradient zone. Conversely, in the limit of a very large gradient zone, the mass of the regions $A$ and $B$ becomes negligible, and the gradient zone has zero net flux. In this limit the pistons undergo the fastest possible motion from left to right. Hence we see that increasing the buffer regions extended the duration of a simulation in two ways. Firstly, it allows the pistons to move a larger distance. Secondly, it reduces the rate of motion.

Differentiating eqn 19 and inserting in eqn 18, we obtain an explicit expression for the flux of component 2:

$$
J_{2}^{x}=\frac{D_{2} \rho_{2}^{\infty}}{L_{G}} \ln \left[\frac{\rho_{2}^{A}-\rho_{2}^{\infty}}{\rho_{2}^{B}-\rho_{2}^{\infty}}\right] .
$$

From eqn 20 it follows that

$$
I_{1}^{G x}=J_{2}^{x}\left(\frac{\rho_{t o t}}{\rho_{2}^{\infty}}-1\right)
$$

Finally, the absence of a concentration gradients in regions $A$ and $B$ requires that the diffusion fluxes vanish there, and so it follows from eqn 17 that

$$
I_{1}^{A x}=J_{2}^{x}\left(\frac{\rho_{t o t}}{\rho_{2}^{A}}-1\right), \text { and }
$$




$$
I_{1}^{B x}=J_{2}^{x}\left(\frac{\rho_{t o t}}{\rho_{2}^{B}}-1\right)
$$

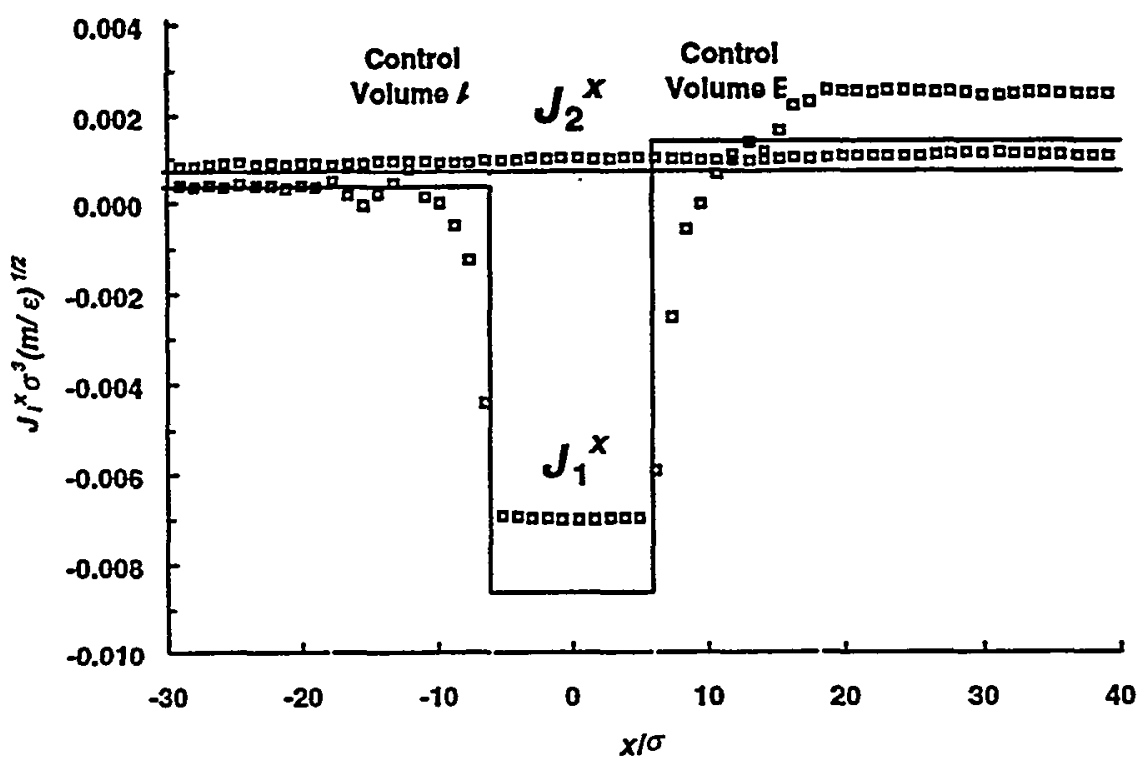

Figure 15. Comparison of the average absolute flux profiles from the constant pressure color diffusion simulation (squares) with the results of the continuum model (solid lines).

In order to compare these predictions for the fluxes against the simulation fluxes, we need to assign values to the parameters $L_{A}, L_{B}, L_{G}, \rho_{t o t}, \rho_{2}{ }^{A}, \rho_{2}{ }^{B}$ and $D_{2}$. The value of the diffusion coefficient was estimated by a method described below. Other parameters were taken directly from the constant-pressure simulation and are summarized in Table 5. The resultant fluxes are plotted in Figure 15, together with the original simulation data. The fluxes in the three different regions are in qualitative agreement with the simulation data, although quantitative agreement was not achieved. This is to be expected, given the many simplifying assumptions, but it validates the underlying principle of the model, which is that the evolution of the simulation is controlled by Fick's law and conservation of momentum.

Table 5. Constant-pressure color diffusion continuum model parameters

\begin{tabular}{|c|c|c|c|c|c|c|}
\hline $\mathrm{D}_{2}(\mathrm{~m} / \varepsilon)^{1 / 2} / \sigma$ & $\sigma^{3} \rho_{2}{ }^{A}$ & $\sigma^{3} \rho_{2}{ }^{B}$ & $\sigma^{3} \rho_{\text {tot }}$ & $L_{A} / \sigma$ & $L_{B} / \sigma$ & $L_{G} / \sigma$ \\
\hline 0.362 & 0.30 & 0.15 & 0.45 & 30 & 30 & 12 \\
\hline
\end{tabular}


The continuum analysis provides us with the answer to our first question regarding how the diffusion coefficient can be determined from the flux and concentration profiles. The local definition of the diffusion coefficients is provided by eqn 18 , based on the local concentration gradient and local diffusion flux, both of which depend on position. Of course, since the overall density of the system is constant, we hope that the local diffusion coefficient turns out to be independent of position. In order to carry out this analysis, we performed a three-parameter least-squares fit of the concentration profiles in the gradient zone to the exponential form of eqn 19 , and then calculated the concentration gradients analytically. The resulting profiles are shown in Figure 16, along with the diffusion fluxes calculated from eqn 17. It can be seen that the concentration gradients and diffusion fluxes decrease in magnitude across the gradient zone. The resultant diffusion coefficients are shown in Figure 17. The values for both components decrease significantly across the gradient zone. There is a significant decrease in the diffusion coefficients from left to right. This is due to the statistical error in the least-squares fit to the concentration profiles. (By fitting the density profiles so as to minimize the square relative error of the density plus the standard deviation of the diffusion coefficient, a very similar fit is obtained, but with a uniform diffusion coefficient profile.) The average diffusion coefficients are very close in either case. The average values for the straight least squares fit are $D_{1}(\mathrm{~m} / \varepsilon)^{1 / 2} / \sigma=0.357$ and $D_{2}(m / \varepsilon)^{1 / 2} / \sigma=0.366$. The average of these two numbers was used in the continuum model described above.We can obtain almost identical results using a simplified the analysis by invoking the following approximation, which is exact at the point where the concentration profiles cross each other:

$$
\begin{aligned}
J_{1 D}^{x} & =(1 / 2)\left(J_{1}^{x}-J_{2}^{x}\right)-(1 / 2)\left(x_{1}-x_{2}\right)\left(J_{1}^{x}+J_{2}^{x}\right) \\
& \approx(1 / 2)\left(J_{1}^{x}-J_{2}^{x}\right)
\end{aligned} .
$$

As can be seen from Figure 11, $\left|J_{1}^{x}-J_{2}^{x}\right|$ is relatively invariant throughout the gradient zone, having an average value of $\left|J_{1}^{x}-J_{2}^{x}\right| \sigma^{3}(m / \varepsilon)^{1 / 2}=0.0080$. In the case of the constant-volume simulation, $\left|J_{1}^{x}-J_{2}^{x}\right| \sigma^{3}(m / \varepsilon)^{1 / 2}$ was 0.0077 in the central gradient zone and 0.0080 in the periodic gradient zone, giving an average value of 0.0079 .

In Table 6 the transport diffusivities calculated using this simplified analysis as well as those obtained from the least-squares fit to the concentration profiles are compared with those of the constant-volume color diffusion simulation.

The values obtained using the simplified and more detailed analyses of the constantpressure simulation agree well with each other, but were somewhat higher than those from the constant-volume simulations. There are several possible reasons for this. Firstly, the total density in the constant-pressure simulation gradient zone was slightly lower (ca. 1\%) than in the constant volume simulations, which should raise the diffusivity by about $1.5 \%$, based on the correlation of Ruckenstein [36]. Secondly, there are significant statistical 
errors in the diffusivities from both methods, which are difficult to estimate precisely, but are probably on the order of several percent.

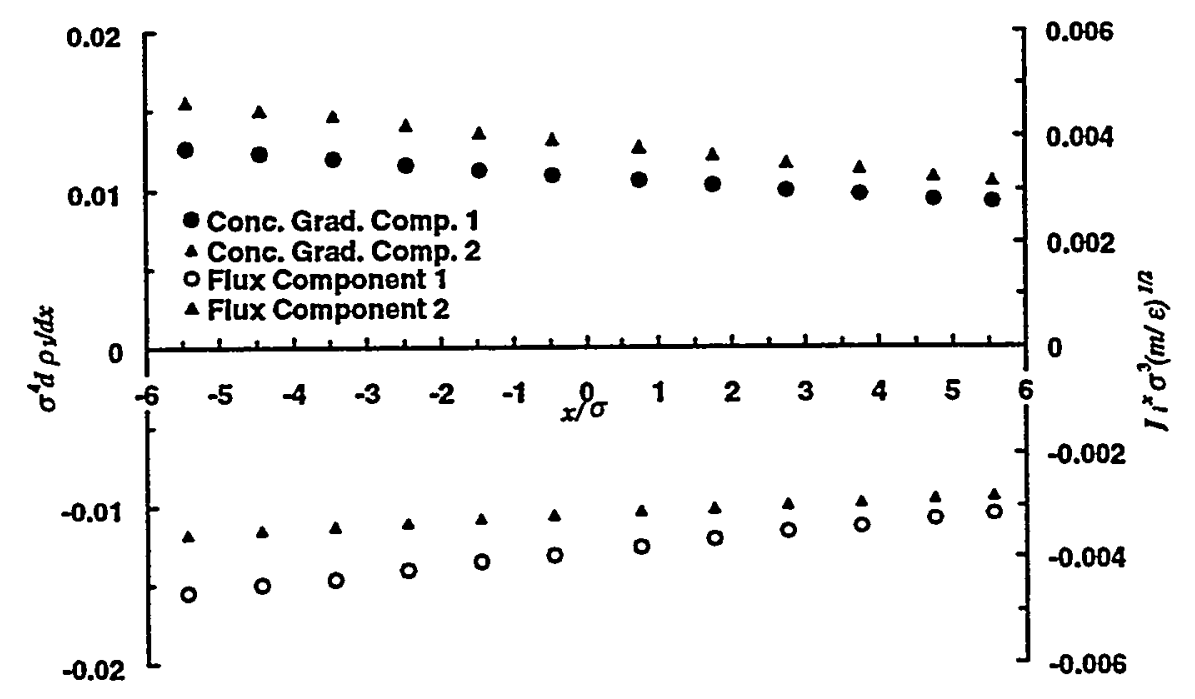

Figure 16. Plot of diffusion fluxes and concentration gradients profiles in the gradient zone from the constant-pressure color diffusion simulation.

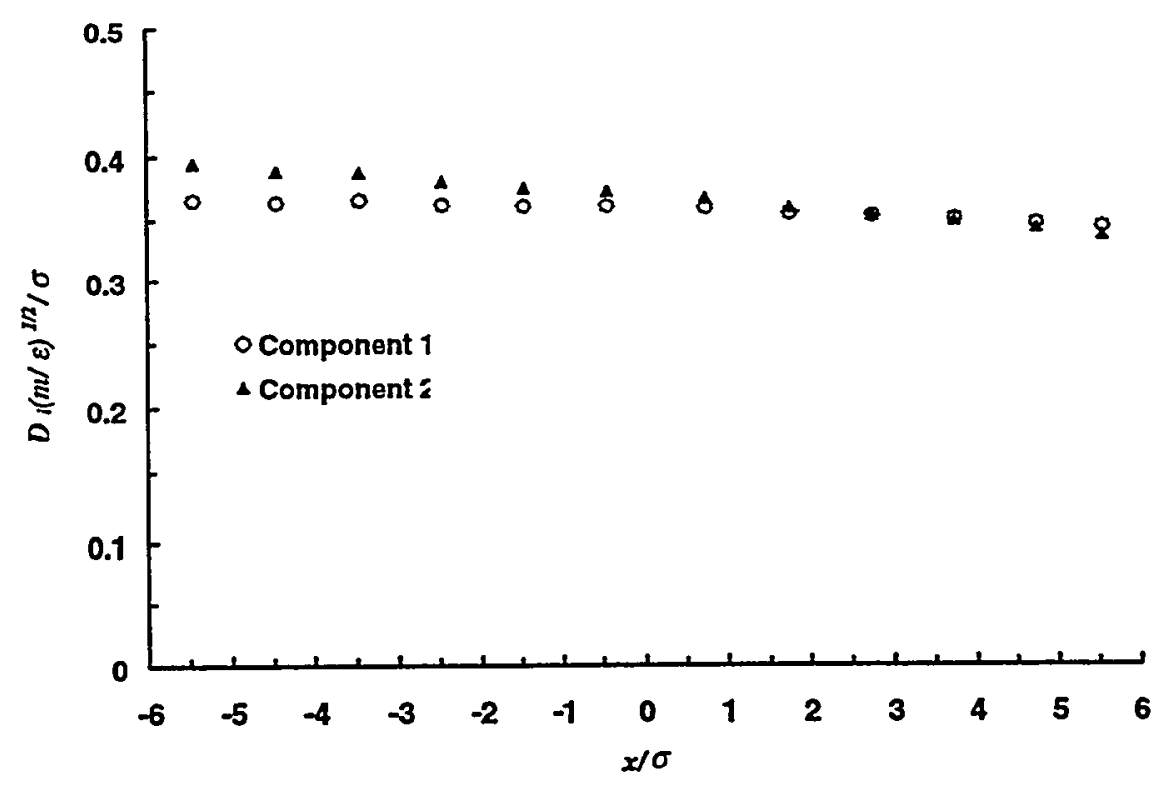

Figure 17. Diffusion coefficient profiles for components 1 and 2 from the constant-pressure color diffusion simulation. 
Gradient-Driven Diffusion of Multi-Atom Molecules Through Macromolecules and Membranes: LDRD 96-0021 Close-out Report

Table 6. Constant-volume and constant pressure simulation results

\begin{tabular}{|c|c|c|c|c|c|c|c|}
\hline & \multicolumn{3}{|c|}{ Constant Volume DCV-GCMD } & \multicolumn{4}{|c|}{ Constant Pressure DCV-GCMD } \\
\hline & & & & \multicolumn{3}{|c|}{ Simplified Analysis } & Detailed \\
\hline Species & $\sigma^{4} \frac{d \rho_{i}}{d x}$ & $\frac{J_{i D}^{x} \sigma^{3}}{\sqrt{\varepsilon / m}}$ & $\frac{D_{i}^{x} \sigma^{-1}}{\sqrt{\varepsilon / m}}$ & $\sigma^{4} \frac{d \rho_{i}}{d x}$ & $\frac{J_{i D}^{x} \sigma^{3}}{\sqrt{\varepsilon / m}}$ & $\frac{D_{i}^{x} \sigma^{-1}}{\sqrt{\varepsilon / m}}$ & $\frac{D_{i}^{x} \sigma^{-1}}{\sqrt{\varepsilon / m}}$ \\
\hline 1 & 0.0124 & 0.00394 & 0.317 & 0.0106 & 0.00399 & 0.376 & 0.357 \\
\hline 2 & 0.0122 & 0.00394 & 0.323 & 0.0109 & 0.00399 & 0.366 & 0.366 \\
\hline Ave. & 0.0123 & 0.00394 & 0.320 & 0.0108 & 0.00399 & 0.371 & 0.362 \\
\hline
\end{tabular}

\section{Binary Diffusion}

As a second test of the constant-pressure method, we applied it to a fairly dense binary mixture of WCA spheres, with a size ratio of 1:2, i.e. $\sigma_{1}=\sigma, \sigma_{2}=2 \sigma$. The Lennard-Jones energy $\varepsilon$ and mass $m$ of both components were the same. As before, both constant volume and constant pressure simulations were performed. The reduced temperature was set to $k T /$ $\varepsilon=1.0$. The chemical potentials used in the constant-volume simulation, as well as the pressure used in the constant-pressure simulation are given in Table 7. These values were chosen to create the following conditions. A volume fraction of 0.3 in control volume $A$ was targeted, with $30 \%$ component 1 by volume. The volume fraction in control volume $B$ was chosen to match the pressure in control volume $A$, with $70 \%$ component 2 by volume. The required volume fraction in control volume $B$ turned out to be 0.22 . The chemical potentials required to create these conditions were calculated using the Carnahan-Starling-Mansoori equation of state for hard sphere mixtures [37]. Effective hard-sphere diameters for the WCA particles were calculated using a correlation due to Ben-Amotz $[36,38]$. The final densities differed from the target values by less than $2 \%$.

Table 7. Thermodynamic conditions of binary diffusion simulations

\begin{tabular}{|l|c|c|c|c|c|c|}
\hline & $\mu_{1}{ }^{\mathrm{A}} / \varepsilon$ & $\mu_{1}{ }^{\mathrm{B}} / \varepsilon$ & $\mu_{2}{ }^{\mathrm{A}} / \varepsilon$ & $\mu_{2}{ }^{\mathrm{B}} / \varepsilon$ & $P \sigma^{3} / \varepsilon$ & $k T / \varepsilon$ \\
\hline Constant $\mathrm{V}$ & 1.007 & 1.267 & 8.886 & 7.219 & - & 1.0 \\
\hline Constant $\mathrm{P}$ & 1.007 & 1.267 & - & - & 0.823 & 1.0 \\
\hline
\end{tabular}

The constant-volume simulation was performed on a Sandia's Intel Teraflop massively parallel computer using an $8 \times 4 \times 4$ processor array. The system extended from $-24 \sigma$ to $24 \sigma$ in the $x$-direction and from $-6 \sigma$ to $6 \sigma$ in the $y$ and $z$ directions. The control volumes were located on the ranges $-18 \sigma<x<-6 \sigma$ and $6 \sigma<x<18 \sigma$. The MD timestep was 
$0.005\left(\varepsilon / m \sigma^{2}\right)^{1 / 2}$. The simulation was started from an empty box and was equilibrated for 500,000 timesteps. The relatively long equilibration time was required in order to ensure that a steady-state had been reached. This was difficult to determine, as the total flux in the system underwent significant large fluctuations in the positive and negative $x$-directions. The average acceptance rates for attempted insertions were 0.1 and 0.001 for components 1 and 2 respectively. To improve the exchange of component 2 , a $10 \% / 90 \%$ selection bias was used when selecting which component to insert or delete. Attempts to use the constant volume method to simulate mixtures with a total volume fraction of 0.5 failed due to extremely low acceptance rates for the large component. After equilibration, the simulation was run for 300,000 timesteps, during which the concentration, chemical potential and flux profiles were sampled.

The constant-pressure simulation was carried out on an $14 \times 4 \times 4$ processor grid in a manner similar to that of the constant-pressure color diffusion simulation described in the previous section. The simulation box extended from $-42 \sigma$ to $+42 \sigma$ in the $x$-direction and from $-12 \sigma$ to $+12 \sigma$ in the $y$-and $z$-directions. The simulation was started from an empty box with fixed walls located at $-40 \sigma$ and $+19 \sigma$. These positions were chosen to be slightly greater than the lowest allowable $x$-coordinates for the left and right pistons, so as to maximize the available range of left-to-right motion, while giving the pistons some room to fluctuate during startup. To quickly equilibrate the system, the control volumes were temporarily extended out to the ends of the simulation box, and both species were inserted and deleted, using the same chemical potentials as before. A stable concentration profile was established after only 75,000 timesteps. This suggests that using fixed walls instead of periodic boundary conditions may stabilize the simulation and dampen fluctuations. From this configuration, a short constant pressure run was conducted, in order to estimate the rate of motion of the pistons. Then a constant-pressure simulation was executed for 300,000 timesteps. The pressure was set at $P \sigma^{3} / \varepsilon=0.823$ (equal to the average pressure from the constant-volume simulation). The control volumes coordinates were reset to those used in the constantvolume simulation.

In Figure 18 we compare the concentration profiles obtained from the two simulations. The agreement in control volume $A$ is good, but in control volume $B$, the constant-pressure simulation seems to be have a small gradient. This is probably due to an insufficient rate of insertion of component 1 , which tends to diffuse out of the left side of the control volume. In all cases, the profiles in the gradient zone exhibit slight non-linearity.

The flux profiles from both simulations are shown in Figure 19. The constant-volume simulation exhibits an asymmetry in the fluxes; component 1 diffuses nearly ten times faster than component 2 . The perfect symmetry introduced by the periodic boundary conditions allow the two components to diffuse at different rates without violating the zero total momentum constraint. Instead, the momentum of each component sums to zero; the average value of the component fluxes is only about $1 \%$ of the value in either gradient zone. The constant-pressure fluxes are qualitatively identical to those from the constant-pressure color diffusion simulation. The flux of component 2 is small and positive throughout. Component 1 is large and negative in the gradient zone, and positive on the left and right. 
Gradient-Driven Diffusion of Multi-Atom Molecules Through Macromolecules and Membranes: LDRD 96-0021 Close-out Report

The total flux sums roughly to zero over the entire profile, which is to be expected, by conservation of momentum.

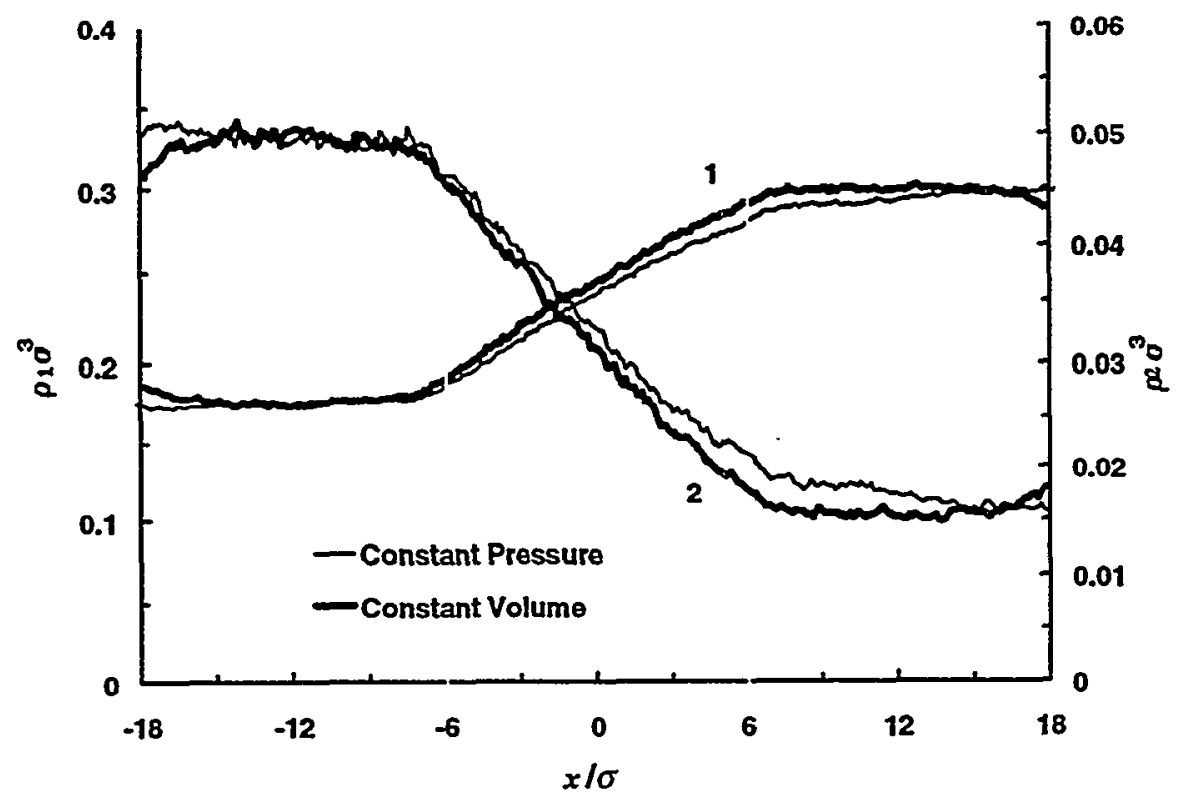

Figure 18. Concentration profiles from simulations of gradient-driven diffusion in a binary mixture of WCA spheres with a 1:2 size ratio. Component 1 is plotted on the left axis and component 2 on the right axis. The heavy lines are from the constant volume simulation, and thin lines are from the constant pressure simulation.

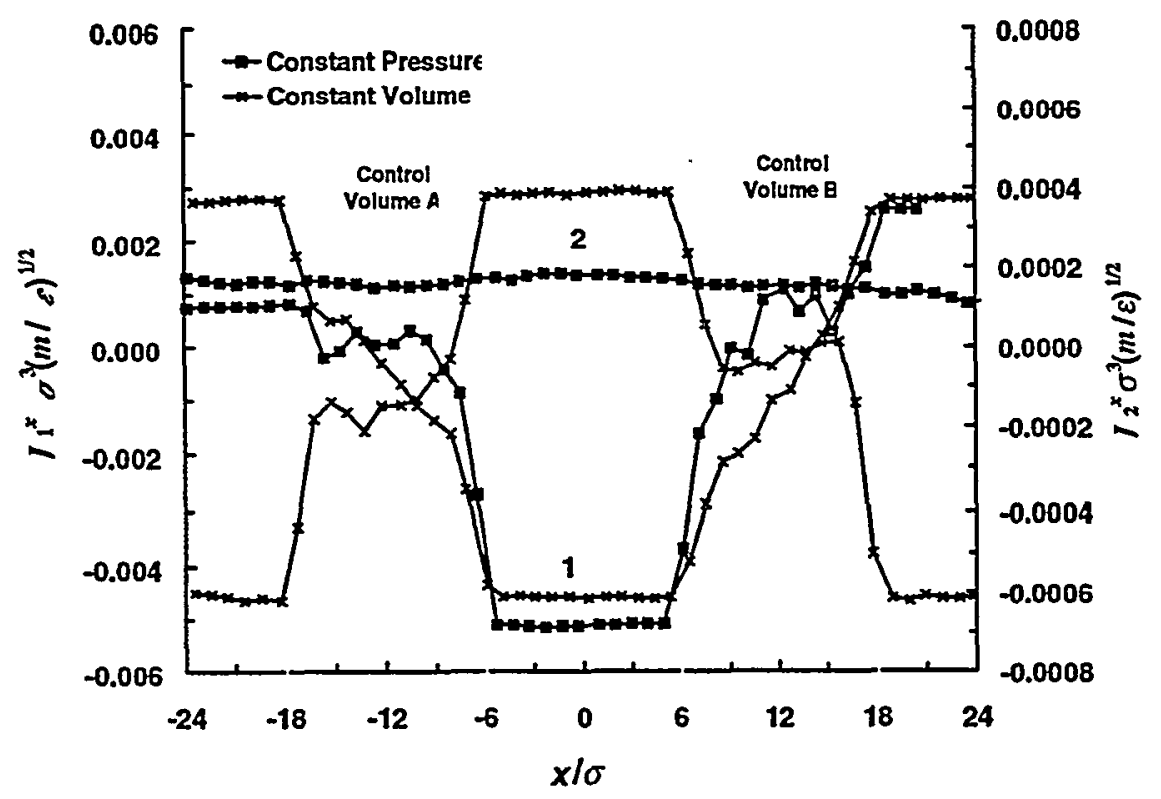

Figure 19. Flux profiles from simulations of gradient-driven diffusion in a binary mixture of WCA spheres with a 1:2 size ratio. Component 1 is plotted on the left axis and component 2 on the right axis. The crosses are from the constant volume simulation, and the squares are from the constant pressure simulation. 
The local binary diffusion coefficients were calculated by the detailed method described in the previous section and the resultant $D_{i}$ profiles are shown in Figure 20. The agreement between the two simulation methods is excellent. $D_{1}$ is smaller than $D_{2}$ by a factor of 4 , due to the differences in the concentration gradients of the two components.

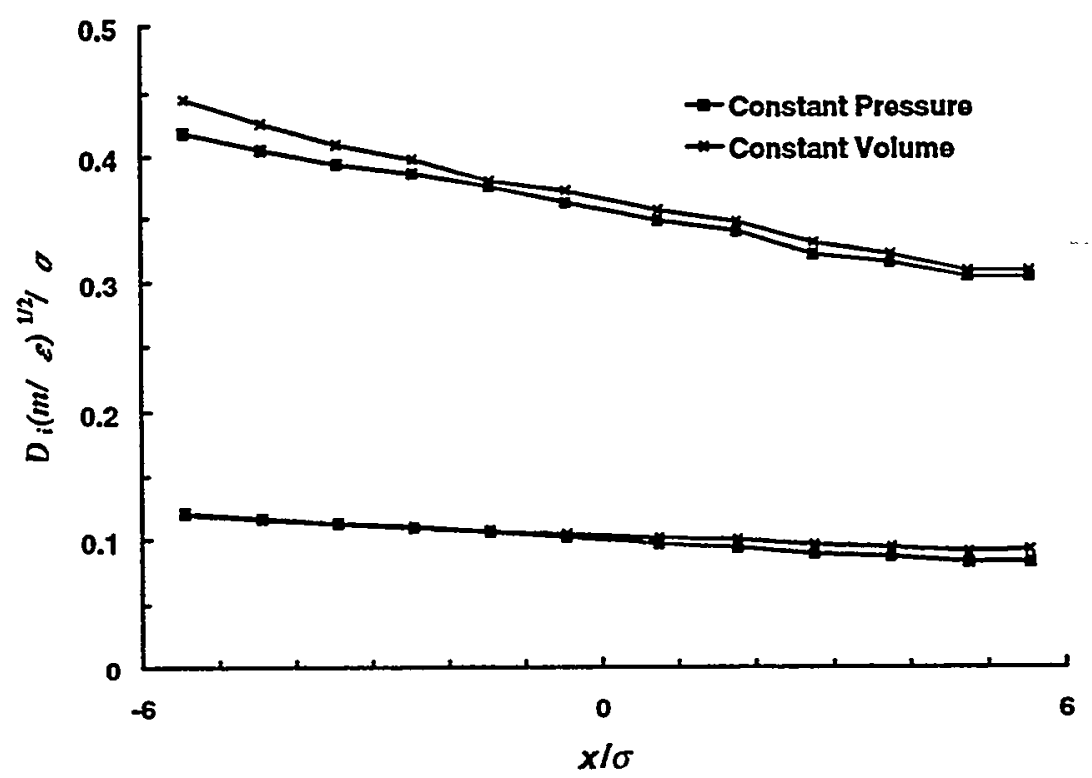

Figure 20. Binary diffusion coefficient profiles from simulations of gradient-driven diffusion in a binary mixture of WCA spheres with a 1:2 size ratio. The lower curves are for component 1 and the upper curves for component 2 . The crosses are from the constant volume simulation, and the squares are from the constant pressure simulation.

Alternatively, we can use the Maxwell-Stefan diffusion coefficients, which are defined by [35]:

$$
\begin{aligned}
& J_{1 D}^{x}=-D_{12} \rho_{1} \beta \frac{d \mu_{1}}{d x} \\
& J_{2 D}^{x}=-D_{21} \rho_{2} \beta \frac{d \mu_{2}}{d x}
\end{aligned}
$$

In order to calculate the chemical potential gradients, we fit the measured chemical potential profiles to the same exponential form used for the density gradients. The resultant diffusivity profiles are shown in Figure 21 . The constant-volume profiles for $D_{12}$ and $D_{21}$ are mirror images of each other. In fact, if the Gibbs-Duhem relation holds, which it should for sufficiently weak gradients, $D_{12}$ and $D_{21}$ should be equal at all positions. The fact that they are not is due to statistical error in the chemical potential gradients. By including a 
Gradient-Driven Diffusion of Multi-Atom Molecules Through Macromolecules and Membranes: LDRD 96-0021 Close-out Report

penalty function based on $\left|D_{12}-D_{21}\right|$ in the fitting process, a slightly different fit is obtained, which satisfies Gibbs-duhem closely, and gives the same average value for $D_{i j}$. Using constant-pressure simulation, only $D_{12}$ can be calculated, as we do not try to measure $\mu_{1}$. The profile in this case is more uniform, and is significantly lower than the average diffusion coefficient from the constant volume simulation.

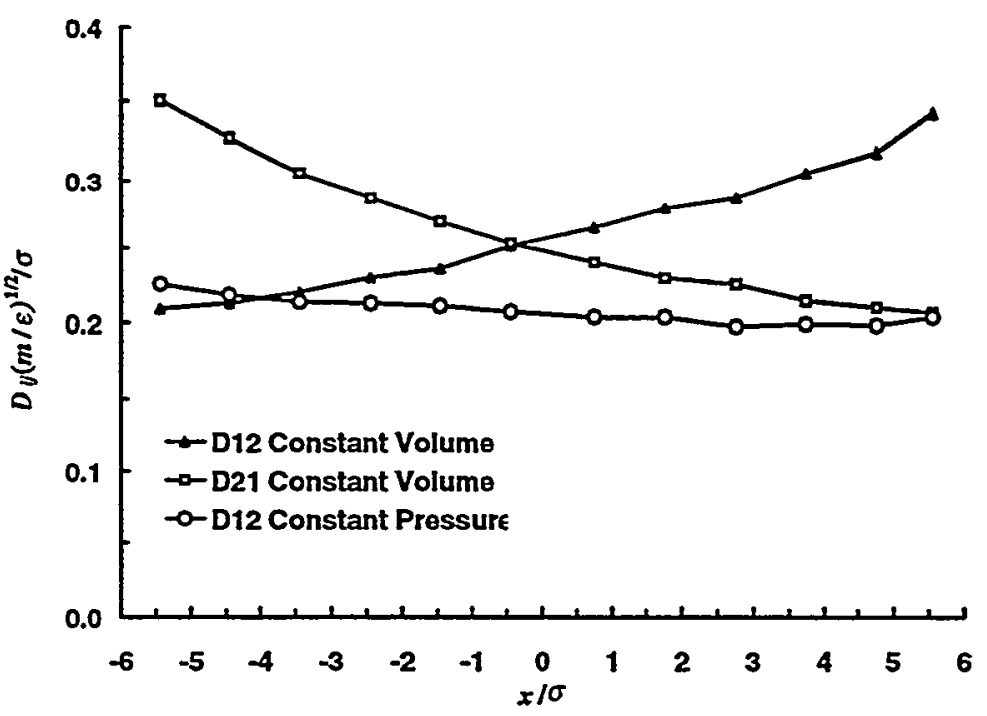

Figure 21. Maxwell-Stefan diffusion coefficient profiles from simulations of gradientdriven diffusion in a binary mixture of WCA spheres with a 1:2 size ratio.

\section{Conclusions}

Since the development of the dual control volume grand canonical method, it has been prototyped it on a variety of systems. Furthermore, while the method was originally developed at Sandia, others, primarily university researchers, have been developing their own DCV-GCMD codes and versions of the method. The general area of simulating systems with chemical potential gradients is becoming an active field.

However, with the extension of the LADERA code to bonded systems, the primary focus of this work, as well as the development of the necessary companion parallel algorithms for the method, Sandia is uniquely positioned to attack the most important and interesting systems where the presence of a chemical potential gradient dominates.

\section{References}

1. G. S. Heffelfinger and F. van Swol, J. Chem. Phys., 100, 7548 (1994).

2. G. S. Heffelfinger and D. M. Ford, Mol. Phys., 94, 659 (1998). 
3. J. M. D. MacElroy, J. Chem. Phys., 101, 5274 (1994).

4. A. P. Thompson, D. M. Ford, and G. S. Heffelfinger, J. Chem. Phys., 109, 1 (1998).

5. M. P. Allen and D. J. Tildesley, Computer Simulation of Liquids, (Clarendon Press, Oxford, 1987).

6. D. Brown and J. H. R. Clarke, Mol. Phys. 51, 1243 (1984).

7. W. G. Hoover, Phys. Rev. A, 31, 1695 (1985).

8. S. J. Plimpton, J. Comp. Phys., 117, 1 (1995), and references therein.

9. G. Heffelfinger and M. E. Lewitt, J. Comp. Chem., 17, 250 (1996).

10. F. van Swol and G. S. Heffelfinger, Mat. Res. Soc. Symp. Proc., 408, 299 (1996).

11. D. M. Ford and G. S. Heffelfinger, Mol. Phys., 94, 673 (1998).

12. S. Plimpton, R. Pollock, and M. Stevens, Proc. of 8th SIAM Conference on Parallel Processing for Scientific Computing (1997).

13. G. S. Heffelfinger, P. I. Pohl, and L. J. D. Frink, Mat. Res. Soc. Symp. Proc., 366, 225 (1995).

14. P. I. Pohl, G. S. Heffelfinger, and D. M. Smith, Mol. Phys. 89, 1725 (1996).

15. P. I. Pohl and G. S. Heffelfinger, Mat. Res. Soc. Symp. Proc., 464, 339 (1997).

16. S. Furukawa, T. Shigeta, and T. Nitta, J. Chem. Engr. of Japan, 29, 725 (1996).

17. S. Furukawa, and T. Nitta, J. Chem. Engr. of Japan, 30, 116 (1997).

18. R. F. Cracknell, D. Nicholson, and N. Quirke, Phys. Rev. Lett., 74, 2463 (1995).

19. D. Nicholson, R. Cracknell, and N. Quirke, Langmuir, 12, 4050 (1996).

20. S. Sunderrajan, C. K. Hall, B. D. Freeman, J. Chem. Phys., 105, 1621 (1996).

21. D. M. Ford and G. S. Heffelfinger, American Physical Society Annual Meeting, Kansas City, MO, 17-21 March (1997)

22. J. H. Thurtell and G. W. Thurtell, J. Chem. Phys., 88, 6641 (1988).

23. W. Dong and H. Luo, Physical Review E, 52, 801 (1995).

24. J. J. Erpenbeck and W. W. Wood, in Statistical Mechanics, Part B: Time-dependent Processes, edited by B. J. Berne, Modern Theoretical Chemistry Vol. 6 (Plenum, New York, 1977).

25. S. Fritzsche, R. Haberlandt, and J. Kärger, Zeitschrift für Physikalische Chemie, 189, 221 (1995).

26. J. W. Evans, M. H. Abbasi, and A. Sarin, J. Chem. Phys., 72, 2967 (1980).

27. D. M. Smith, AIChE Journal, 32, 329 (1986).

28. M. Sun and C. Ebner, Phys. Rev. A, 46, 4813 (1992).

29. D. M. Ford and E. D. Glandt, J. Phys. Chem., 99, 11543 (1995). 
Gradient-Driven Diffusion of Multi-Atom Molecules Through Macromolecules and Membranes: LDRD 96-0021 Close-out Report

30. S. K. Kjelstrup and B. Hafskjold, Ind. Eng. Chem. Res., 35, 4203 (1996).

31. M. Lupkowski and F. van Swol, J. Chem. Phys., 93, 737 (1990).

32. I. C. Sanchez, and U. Bidkar, Bull. Amer. Phys. Soc., Ser. II, 42, No. 1, (1997).

33. S. J. Plimpton, and J.D. McCoy, private communication.

34. A. P. Thompson, and G. S. Heffelfinger, in preparation, (1998).

35. R. Taylor, and R. Krishna, Multicomponent Mass Transfer, (Wiley, 1993).

36. E. Ruckenstein and H. Liu, Ind. Eng. Chem. Res., 36, 3927 (1997)

37. T. M. Reed and K. E. Gubbins, "Applied Statistical Mechanics", Butterworth-Heinemann (1991)

38. D. Ben-Amotz and D. R. Herschbach, J. Phys. Chem., 94, 4714 (1990) 


\section{DISTRIBUTION:}

1 MS-0188 LDRD Office, 4001

1 MS-0321 W. J. Camp, 9200

MS-0441 R. W. Leland, 9226

1 MS-0619 Review \& Approval Desk

for DOE/OSTI

MS-0710 T. M. Nenoff, 1845

MS-0720 P. I. Pohl

MS-0820 P. Yarrington, 9232

MS-0826 K. S. Chen, 9111

2 MS-0899 Technical Library, 4916

MS-1111 S. Dosanjh, 9221

MS-1111 A. P. Thompson, 9225

5 MS-1111 G. S. Heffelfinger, 9225

1 MS-1111 S. J. Plimpton, 9221

MS-1349 F. van Swol, 1841

MS-1411 G. S. Grest, 1843

1 MS-9018 Central Technical Files

David M. Ford

Texas A\&M University

Department of Chemical Engineering

College Station, TX 77843-3122

Marcus G Martin

Department of Chemistry

University of Minnesota

207 Pleasant Street SE

Minneapolis, MN 55455-0431

Brian K. Peterson

Air Products and Chemicals, Inc.

7201 Hamilton Boulevard

Allentown, PA 18195-1501 